\title{
Immunotherapy in non-small-cell lung cancer: a bridge between research and clinical practice
}

\author{
Passiglia Francesco*,1, Commendatore Oriana ${ }^{2}$, Vitali Milena ${ }^{3}$ \& Conca Raffaele 4 \\ ${ }^{1}$ Department of Surgical, Oncological \& Stomatological Disciplines, University of Palermo, 90100 - Palermo, Italy \\ ${ }^{2}$ Medical Oncology Unit, ARNAS Garibaldi, Catania, Italy \\ ${ }^{3}$ Thoracic Oncology Unit, Medical Oncology Department, Istituto Nazionale dei Tumori, Milano, Italy \\ ${ }^{4}$ Division of Medical Oncology, Department of Onco-Hematology, IRCCS-CROB, Referral Cancer Center of Basilicata, 85028 \\ Rionero, Vulture (PZ), Italy. \\ *Author for correspondence: passi.f@live.it
}

\begin{abstract}
Lung cancer has been historically considered a poorly immunogenic disease because of the few evidence of immune responses in affected patients and the limited efficacy of immunomodulating strategies. Recent understanding of the molecular mechanisms leading to cancer immune evasion has allowed the development of a new class of drugs called immune checkpoint inhibitors, which reactivate host responses with outstanding clinical benefits in a portion of patients with non-small-cell lung cancer. In this review, we briefly summarize the basis of immunogenicity and immune escape of cancer, with specific focus on nonsmall-cell lung cancer, mechanisms underlying immune checkpoint inhibitors efficacy and the most updated results on potential biomarkers, with the final aim of defining current unmet needs of immunotherapy in clinical practice.
\end{abstract}

First draft submitted: 1 February 2018; Accepted for publication: 30 May 2018; Published online: 10 July 2018

Keywords: biomarkers $\bullet$ cancer immunogenicity $\bullet$ clinical studies $\bullet$ immune checkpoint inhibitors $\bullet$ NSCLC $\bullet$ PD-1 - PD-L1 • translational research

Lung cancer has been historically considered a poorly immunogenic disease because of the few evidence of immune responses in affected patients and the limited efficacy of immunomodulating strategies, including vaccines [1]. Nevertheless, the recent understanding of the molecular mechanisms leading to cancer immune evasion has allowed to develop a new class of drugs, known as immune checkpoint inhibitors (ICIs), able to reactivate host responses and to mediate outstanding clinical benefit in a sizable proportion of patients with non-small-cell lung cancer (NSCLC).

Until a few years ago, the only therapeutic option for patients with metastatic NSCLC was platinum-based chemotherapy or target therapy for adenocarcinoma subtypes harboring known molecular alterations in driver oncogenes (EGF receptor [EGFR]/anaplastic lymphoma kinase [ALK]) [2]. However, the significant success of ICI in melanoma has paved the way to the clinical testing of this strategy in other solid tumors, including NSCLC [3]. Surprisingly, the stimulation of antitumor immune response through PD-1/PD-L1 blockade resulted in a significant and durable disease control in a subgroup of patients with advanced NSCLC, with a remarkable impact on overall survival (OS) and notable improvement of patient quality of life.

On the basis of these initial results, PD-1/PD-L1 ICIs are currently recommended as new standard treatment for NSCLC patients. Particularly to date, we have three monoclonal antibodies (MoAbs), nivolumab, pembrolizumab and atezolizumab, approved by regulatory authorities in second-line setting, of which one, pembrolizumab is approved also as first-line therapy [4]. The most recent survival update of the CheckMate 003 Phase I study of nivolumab in pretreated NSCLC patients revealed that about $16 \%$ of patients are still alive after 5 years of therapy, suggesting that a small but significant subgroup of patients derive an impressive and durable benefit from ICIs [5]. Conversely, evidence emerging from randomized trials showed that about $50 \%$ of patients do not benefit from treatment [6-9]. 
Thus, the identification of predictive biomarkers is an urgent need, representing one of the main objectives of lung cancer translational research that could help oncologists to select which patients may really benefit from immunotherapy. Unlike target therapies, immunotherapy modulates a complex network of molecular and cellular pathways, making the discovery of predictive biomarkers a very hard process and a challenging task for translational research. In addition, the interaction between tumor and immune system observed in preclinical models may not reflect what occurs in cancer patients, thus it does not necessarily provide reliable information for human use. Hence, the process has to rely mostly on clinical setting and on the availability of biological samples collected in a systematic and standardized manner from defined patients subsets. This means that the scientific community should promote a new era of clinical trials aimed at studying biological samples for the identification of immune-related biomarkers that has to be timely addressed.

With the present review, we would like to briefly summarize the basis of immunogenicity and immune escape in human cancers, with specific focus on NSCLC, the mechanisms underlying ICI clinical efficacy and the most updated results in term of potential biomarkers, with the final aim of defining the current unmet needs of immunotherapy in clinical practice. The broad use of ICI that is presently applied to NSCLC offers oncologists the unique opportunity of actively contributing to these studies, for a bridge between research and clinical practice.

\section{Basis of cancer immunogenicity \& immune escape}

The immune mechanisms leading to the induction of a response against the tumor cells are very complex, but they can be envisaged as the result of opposite forces regulating tumor/host interaction during the different disease phases. From one side, the immunogenicity of cancer cells, in other words, the expression of 'altered' proteins detected by the immune system as 'antigens', induces the priming of antigen-specific T-cells through the processing and presentation by dendritic cells (DC) of antigenic determinants in the context of the human leukocyte antigen (HLA) molecules. Antigenic determinants in NSCLC are provided by tumor proteins ectopically induced by neoplastic transformation (as for cancer/testis antigens) [10], epithelial molecules increased in expression density (EGFR, carcinoembryonic antigen (CEA), epithelial cell adhesion molecule (EpCAM), mucin1 (MUC1)) [11] or mutated proteins deriving from cancer genetic instability that leads to the generation of new amino acid sequences able to be recognized by T-cells as 'neoantigens' [12]. The latter groups, globally quantified by measuring the tumor mutational burden, are considered to provide the most immunogenic epitopes and to associate with the presence of more efficient antitumor immune responses in course of immunotherapy [13].

Upon the engagement of their T-cell receptor (TCR) with the antigen/HLA complex expressed on tumor cells, lymphocytes mediate tumor cell lysis through the release of cytolytic granules (containing perforin, granzyme B and proapoptotic molecules such as FasL and TRAIL) and stimulatory cytokines such as IFN- $\gamma$, IL-2 and TNF- $\alpha$. Sign of this reactivity is the presence of a T-cell infiltrate, most commonly composed by $\mathrm{CD} 8^{+} \mathrm{T}$-cells that is often detected in solid cancers, including NSCLC, as linked to good prognosis [14], indicating that even in patients with clinically evident cancer, an active immune response can contribute in slowing-down disease progression.

Nevertheless, when this immunity is unable to clear the tumor, it drives instead a sort of chronic 'evolutionary' process that causes the selection of immune-resistant tumor cell clones, according to a mechanism known as immunoediting, and including the onset of antigen- or HLA-loss tumor cell variants [15]. Concomitantly, multiple immune escape strategies take place within the tumor microenvironment (TME), mainly relying on two pathways: the upregulation on T-cells of immune checkpoints (PD-1, CTLA-4, TIM3 and LAG3) that, binding to cognate ligands also induced on tumor and stroma cells by IFN- $\gamma$, deliver inhibitory signals to T-cells causing their reversible paralysis (exhaustion) [16]; the accrual at tumor site of immune regulatory cells that exert suppressive activity on $\mathrm{T}$ lymphocytes and block their function in the attempt to maintain tissue homeostasis. To the latter belong regulatory T-cells (Tregs) and the large and heterogeneous family of myeloid-derived suppressor cells (MDSCs). Both cell subsets belong to the physiological process of immune homeostasis and represent the response of the host to chronic inflammation, aimed at contrasting persistent immune stimulation. Specifically, Treg $\left(\mathrm{CD}^{+} \mathrm{CD}^{+}\right.$, $\mathrm{FOXP}^{+}$, CD25 high) are cells devoted to control autoimmunity [17], and they result increased in number and activation state in blood, lymph nodes and tumor site of cancer patients, including NSCLC [18]. They exert immunosuppression on effector $\mathrm{CD} 8^{+} \mathrm{T}$-cells through multiple pathways including blocking of IL-2 synthesis, release of T-cell inhibitory cytokines such as IL-10 and TGF- $\beta$, and high expression of CTLA- 4 . Tregs restrain T-cell activation and their depletion is associated with more effective and durable response to immunotherapy, particularly to CTLA-4 blockade [19]. 


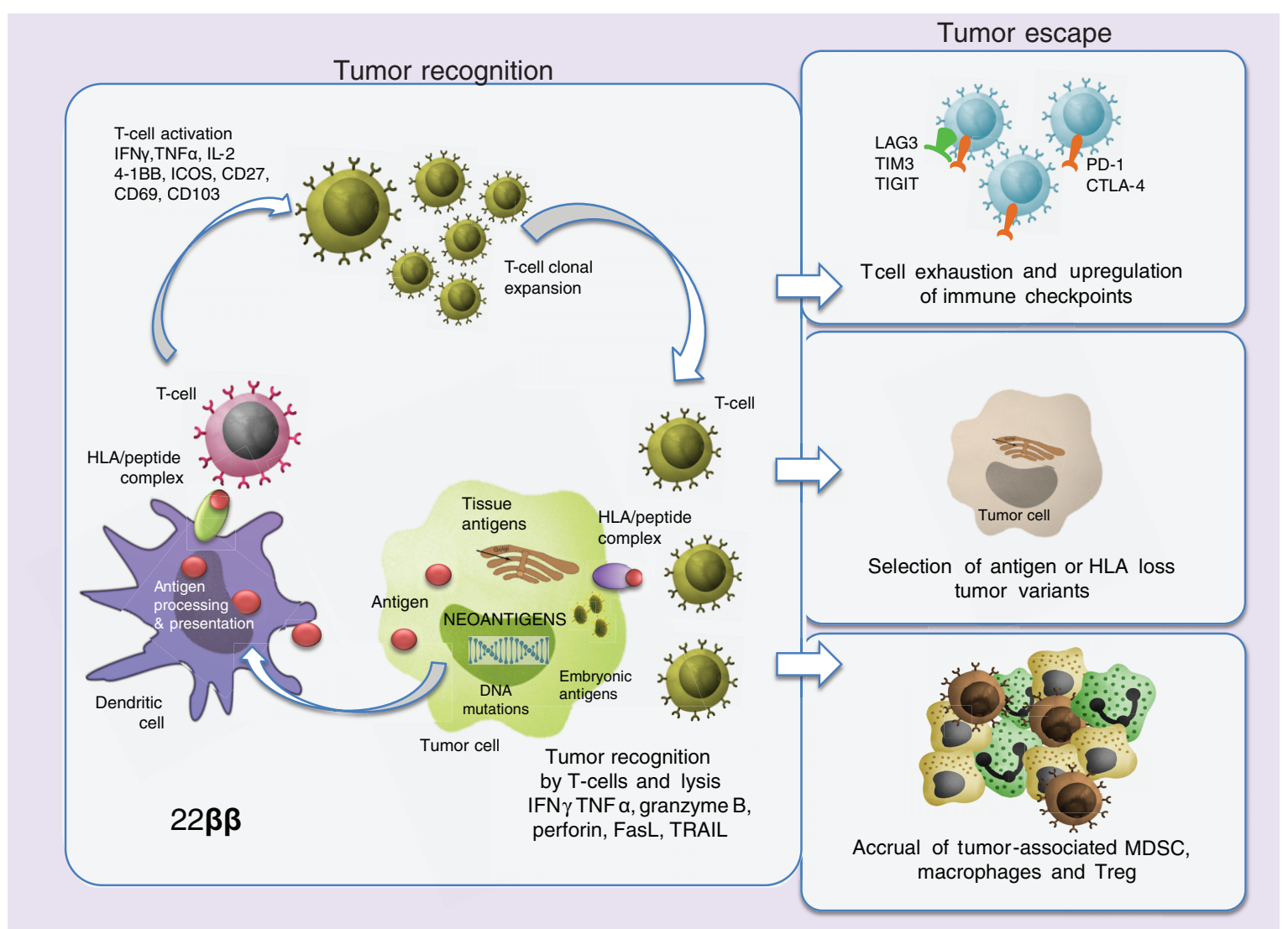

Figure 1. Basis of cancer immunogenicity and immune escape.

NSCLC lesions are also enriched in myeloid cells with respect to nontumor surrounding lung tissue, including cells of monocytic, granulocytic and macrophagic phenotype [18,20]. Aside from residential alveolar macrophages, most of these elements derive from the differentiation of immature myeloid cells coming from the blood and globally defined as MDSC [21]. It is indeed largely acknowledged that chronic immune stimulation, together with different cytokines and chemokines secreted by tumor cells, molds bone marrow myelopoiesis and promotes the mobilization of late precursors in the attempt to restrain inflammation and maintain tissue homeostasis. Pathological accrual of MDSC in blood, lymph nodes and TME of cancer patients, reproducibly occurring in association with poor prognosis [22] and limited response to immunotherapy, causes potent suppression of antitumor T-cells (via TGF- $\beta$, reactive oxygen species, nitric oxide synthase and indoleamine 2,3-dioxygenase [IDO]), stroma remodeling, VEGF-mediated angiogenesis and metastatic spread. These cells, defined as monocytic (CD14 ${ }^{+}$HLA-DRneg) or granulocytic (CD15 HLA-DRneg) MDSC by cytofluorimetry, are measured as monocytes and granulocytes in routine blood test and this explains the negative prognostic impact that neutrophil or monocyte absolute counts, together with the relative ratio with lymphocytes, play in patients with cancer, including NSCLC [23,24] (Figure 1).

This complex immune scenario featuring TME has been hard to modulate in a consistent and pleiotropic fashion until few years ago, when immune checkpoints and their role in favoring tumor immune escape were discovered. CTLA-4 and PD-1, together with the cognate ligand PD-L1, are indeed the common denominators of many of the multiple immune cell subsets present in TME whose modulation, through antagonist MoAbs, has been recently shown to tilt the immune balance toward antitumor immune responses that can control disease progression in a substantial percentage of patients.

\section{Mechanisms of action of PD-1 \& PD-L1 inhibitors}

CTLA-4 and PD-1 are two inhibitory receptors expressed on T-cells that, binding to their ligands (CD80/CD86 and PD-L1/PD-L2, respectively) expressed on several types of immune and tumor cells [25,26], play a central role in suppression of T-cell activity, thus regulating immune-response against tumor cells. CTLA-4 acts in the 
activation stage of T-cell priming, which occurs in regional secondary lymphoid organs and is necessary to generate a population of effector T-cells against the tumor. On the other hand, PD-1 acts in the secondary stage of T-cell activation, binding its ligands on tumor cells and other stromal cells of TME [27,28]. In the last 10 years, many clinical trials on antibodies against CTLA-4, PD-1 and PD-L1 have shown encouraging antitumor activity in various malignancies, including NSCLC [22,29-33]. However, despite this major progress, many questions remain regarding the optimal use of ICI, as only approximately $30 \%$ of patients seem to benefit from this approach [34].

The blockade of PD-1 receptor can restore the activity of chronically exhausted tumor-specific T-cells. After binding with its natural ligands - PD-L1 (also known as B7-H1 or CD274) and PD-L2 (also known as B7-DC or CD273) - PD-1 receptor can negatively regulate the immune system, promoting self-tolerance and allowing tumor cells to escape from immune control [35]. PD-1 is a 288-aminoacid type-1 transmembrane protein of the immunoglobulin superfamily, encoded by the five exon Pdcd1 gene located on chromosome 1 in mice and chromosome 2 in humans and normally expressed on B cells, T-cells and monocytes [36-38]. After interaction with its ligands, the intracellular domain of PD- 1 is phosphorylated, leading to the downmodulation of the TCR signaling [39,40] and to a limitation of T-cell activity in the TME [41]. Moreover, also CD80 or B7-1 complex has been shown to bind PD-1, inducing inhibitory signals in T-cells with a subsequent anergic state $[42,43]$. The centrality of PD-1 in the immune regulation after chronic tumor antigen stimulation was confirmed by the first demonstration of PD-1 high expression on lymphocytes infiltrating human tumors activated by melanoma specific antigens, as reported by Fourcade et al. in 2009, and by its high presence on Treg cells that are among the most important CD4 ${ }^{+}$ T-cells with suppressive function [44-50]. PD-L1 is the principal ligand of PD-1 receptor and is expressed on activated hematopoietic and epithelial cells, while PD-L2 is more present on DC and macrophages [26]. Immunohistochemical (IHC) assays have revealed high PD-L1 upregulation on cells of many different tumors including NSCLC [35]. Expression of PD-L1 on tumor cells is dependent on two mechanisms: an intrinsic expression correlated to various aberrant oncogenic signal pathways, such as phosphoinositide 3-kinase/protein kinase B, extracellular-signalregulated kinases/mitogen-activated protein kinase and Janus kinase (JAK2)/STAT, and an induced expression dependent on inflammatory signals such as IFN- $\gamma$ release induced by active antitumor immune response [32,51-54]. The adaptive mechanism seems to be more frequently involved in PD-L1 expression in TME, suggesting that the presence of PD-L1 is directly correlated to a previous activation of T-cells specific for a tumor antigen [55]. High PD-L1 expression was demonstrated on melanoma, ovarian, lung and other types of cancer cells [56-63]. Moreover, high PD-L1 expression was associated with strong presence of tumor-infiltrating lymphocytes (TILs) and IFN- $\gamma$ in melanoma TME, explaining how activation of PD-L1/PD-1 pathway is an immune-resistance mechanism used by cancer cells to escape from immune-related destruction [53]. This can explain the rationale to use PD-1/PD-L1 ICIs in the treatment of tumors expressing high levels of PD-L1, with the aim to block tumor-induced immune suppression. In fact, MoAbs directed against PD-1 or PD-L1 can block T-cell inhibition mediated by PD-1 pathway, restoring immune response; many preclinical studies demonstrated the antitumor activity of these antibodies in murine tumor models, leading to further investigation in cancer patients [50,64]. Moreover, in preclinical setting, it has been demonstrated that also the Fc-gamma receptors of anti-CTLA-4 play a role in vivo models, increasing $\mathrm{CD}^{+}$to Treg cell ratio. These data suggest that, in highly immunogenic tumors, also the Fc-gamma receptors polymorphism status could be considered in the selection of patients likely to respond to anti-CTLA-4 [19].

\section{ICls in advanced NSCLC}

On the basis of encouraging activity observed in early clinical trials, several MoAbs targeting PD-1 and PD-L1 have been investigated in clinical setting, showing a great clinical benefit in several tumor types, including NSCLC (Table 1).

Nivolumab (IgG4 anti-PD-1 MoAb) is the first ICI evaluated for safety and clinical activity in a Phase I doseescalation expansion cohort study in patients with advanced heavily pretreated NSCLC. For all 129 patients, treated with different doses, median OS was 9.9 months (14.9 months in patients receiving nivolumab $3 \mathrm{mg} / \mathrm{kg}$ ) and overall response rate (ORR) was $17 \%$; squamous and nonsquamous histologies had similar efficacy [66]. This benefit was confirmed in two Phase III randomized trials (CheckMate 017 trial for squamous and CheckMate 057 trial for nonsquamous NSCLC). According to these findings, nivolumab was the first human anti-PD-1 MoAb approved by the US FDA for treatment of patients with metastatic both squamous and nonsquamous NSCLC who progressed after first-line therapy.

Pembrolizumab is a humanized monoclonal IgG4-K isotype antibody against PD-1. In a Phase I expansion cohort trial (Keynote 001 trial) of pretreated and not-pretreated patients with advanced NSCLC, pembrolizumab, 


\begin{tabular}{|c|c|c|c|c|c|c|c|}
\hline Agent & Description & $\begin{array}{l}\text { NSCLC setting } \\
\text { approvation }\end{array}$ & Trial & $\begin{array}{l}\text { N. patients } \\
\text { randomized }\end{array}$ & Outcomes & TrAE (G3-4) n. (\%) & $n(\%)$ of TrAEs \\
\hline \multirow[t]{2}{*}{ Nivolumab } & \multirow[t]{2}{*}{$\begin{array}{l}\text { Fully human } \\
\text { anti-PD-1 IgG4 } \\
\text { monoclonal } \\
\text { antibody }\end{array}$} & $\begin{array}{l}\text { Stage IIIB or IV } \\
\text { squamous-cell NSCLC } \\
\text { after treatment with } \\
\text { platinum-based } \\
\text { chemotherapy }\end{array}$ & $\begin{array}{l}\text { CheckMate } 017 \text { [7] } \\
\text { Phase III } \\
\text { Nivolumab } 3 \mathrm{mg} / \mathrm{kg} \\
\text { q2w vs docetaxel } \\
75 \mathrm{mg} / \mathrm{mq} \mathrm{q} 3 \mathrm{w}\end{array}$ & 272 & $\begin{array}{l}\text { OS: } 9.2 \text { vs } 6.0 \mathrm{~m} \text {; HR: } \\
\text { 0.59; } p<0.001 \\
\text { RR: } 20 \text { vs } 9 \% ; p=0.008 \\
\text { PFS: } 3.5 \text { vs } 2.8 \mathrm{~m} ; \mathrm{HR}: \\
0.62 ; p<0.001\end{array}$ & $\begin{array}{l}\text { All } \\
\text { Fatigue } \\
\text { Nausea } \\
\text { Anorexia } \\
\text { Pneumonitis } \\
\text { Hypothyroid } \\
\text { Diarrhea }\end{array}$ & $\begin{array}{l}58(7) \\
16(1) \\
9(0) \\
11(1) \\
5(0) \\
1(0) \\
8(0)\end{array}$ \\
\hline & & $\begin{array}{l}\text { Stage IIIB or IV } \\
\text { nonsquamous lung } \\
\text { cancer after treatment } \\
\text { with platinum-based } \\
\text { chemotherapy and a } \\
\text { tyrosine kinase } \\
\text { inhibitor for } \\
\text { EGFR/ALK-mutated } \\
\text { patients }\end{array}$ & $\begin{array}{l}\text { CheckMate } 057 \text { [6] } \\
\text { Phase III } \\
\text { Nivolumab } 3 \mathrm{mg} / \mathrm{kg} \\
\text { q2w vs docetaxel } \\
75 \mathrm{mg} / \mathrm{mq} \mathrm{q3w}\end{array}$ & 582 & $\begin{array}{l}\text { OS: } 12.2 \text { vs } 9.4 \mathrm{~m} ; \quad H R: \\
0.73 ; p=0.002 \\
\text { RR: } 19.2 \text { vs } 12.4 \% ; \quad p \\
=0.02 \\
\text { PFS: } 2.3 \text { vs } 4.2 \mathrm{~m} ; \mathrm{HR}: \\
0.92 ; p=0.39\end{array}$ & $\begin{array}{l}\text { All } \\
\text { Fatigue } \\
\text { Nausea } \\
\text { Anorexia } \\
\text { Pneumonitis } \\
\text { Hypothyroid } \\
\text { Diarrhea }\end{array}$ & $\begin{array}{l}69(10) \\
16(1) \\
12(1) \\
10(0) \\
1(1) \\
7(0) \\
8(1)\end{array}$ \\
\hline Pembrolizumab & $\begin{array}{l}\text { Humanized } \\
\text { anti-PD-1 lgG4-j } \\
\text { isotype } \\
\text { monoclonal } \\
\text { antibody }\end{array}$ & $\begin{array}{l}\text { Advanced NSCLC after } \\
\text { platinum containing } \\
\text { chemotherapy or a } \\
\text { tyrosine kinase } \\
\text { inhibitor for } \\
\text { EGFR/ALK-mutated } \\
\text { patients, with at least } \\
1 \% \text { of tumors } \\
\text { expressing PD-L1 }\end{array}$ & $\begin{array}{l}\text { Keynote } 010[8] \\
\text { Phase II/III } \\
\text { Pembrolizumab } \\
2 \mathrm{mg} / \mathrm{kg} \text { vs } \\
\text { pembrolizumab } \\
10 \mathrm{mg} / \mathrm{kg} \text { vs } \\
\text { docetaxel } \\
75 \mathrm{mg} / \mathrm{mq} \mathrm{q} 3 \mathrm{w}\end{array}$ & 1034 & $\begin{array}{l}\text { Pemb } 2 \mathrm{mg} / \mathrm{kg} \\
\text { OS: } 10.4 \text { vs } 8.5 \mathrm{~m} \text {; } \\
\text { HR: } 0.71 ; p=0.0008 \\
\text { PFS: } 3.9 \text { vs } 4 \mathrm{~m} ; \\
\text { HR: } 0.88 ; p=0.07 \\
\text { Pemb } 10 \mathrm{mg} / \mathrm{kg} \text { OS: } \\
12.7 \text { vs } 8.5 \mathrm{~m} ; \mathrm{HR}: \\
0.61, p<0.0001 \\
\text { PFS: } 4.0 \text { vs } 4.0 \mathrm{~m} ; \mathrm{HR}: \\
\text { O.79; } p=0.004 \\
\text { RR: } 18 \% \text { vs } 18 \% \text { vs } \\
9 \%(p<0.001)\end{array}$ & $\begin{array}{l}\text { All } \\
\text { Fatigue } \\
\text { Nausea } \\
\text { Anorexia } \\
\text { Pneumonitis } \\
\text { Hypothyroid } \\
\text { Diarrhea } \\
\text { All } \\
\text { Fatigue } \\
\text { Nausea } \\
\text { Anorexia } \\
\text { Pneumonitis } \\
\text { Hypothyroid } \\
\text { Diarrhea }\end{array}$ & $\begin{array}{l}63(13) \\
14(1) \\
11(1) \\
14(1) \\
5(2) \\
8(0) \\
7(1) \\
66(16) \\
14(2) \\
9(1) \\
10(1) \\
4(2) \\
8(0) \\
6(0)\end{array}$ \\
\hline Atezolizumab & $\begin{array}{l}\text { Human } \\
\text { anti-PD-L1 } \\
\text { monoclonal } \\
\text { IgG1 antibody }\end{array}$ & $\begin{array}{l}\text { Stage IIIB or IV after } \\
\text { one or two previous } \\
\text { cytotoxic } \\
\text { chemotherapy } \\
\text { regimens (one or more } \\
\text { platinum-based } \\
\text { combination therapies) }\end{array}$ & $\begin{array}{l}\text { POPLAR trial [65] } \\
\text { Phase II } \\
\text { Atezolizumab } \\
1200 \mathrm{mg} \text { vs } \\
\text { docetaxel } \\
75 \mathrm{mg} / \mathrm{mq} \mathrm{q} 3 \mathrm{w}\end{array}$ & 287 & $\begin{array}{l}\text { OS: } 12.6 \text { vs } 9.7 \mathrm{~m} ; \mathrm{HR}: \\
0.73 ; \mathrm{p:} 0.04\end{array}$ & $\begin{array}{l}\text { All } \\
\text { Fatigue } \\
\text { Nausea } \\
\text { Anorexia } \\
\text { Pneumonitis } \\
\text { Hypothyroid } \\
\text { Diarrhea }\end{array}$ & $\begin{array}{l}67(40) \\
20(14) \\
12(8) \\
17(12) \\
- \\
- \\
7(5)\end{array}$ \\
\hline & & & $\begin{array}{l}\text { OAK trial [9] } \\
\text { Phase III } \\
\text { Atezolizumab } \\
1200 \mathrm{mg} \text { vs } \\
\text { docetaxel } \\
75 \mathrm{mg} / \mathrm{mq} \mathrm{q3w}\end{array}$ & 850 & $\begin{array}{l}\text { OS: } 13.8 \text { vs } 9.6 \mathrm{~m} \text {; HR: } \\
0.73 ; \mathrm{p}=0.0003 \\
\text { PFS: } 2.8 \text { vs } 4.0 \mathrm{~m} \text {; HR: } \\
0.95 ; \\
\text { RR: } 14 \text { vs } 13 \%\end{array}$ & $\begin{array}{l}\text { All } \\
\text { Fatigue } \\
\text { Nausea } \\
\text { Anorexia } \\
\text { Pneumonitis } \\
\text { Hypothyroid } \\
\text { Diarrhea }\end{array}$ & $\begin{array}{l}64(37) \\
26(2) \\
17(0) \\
23(0) \\
- \\
- \\
15(0)\end{array}$ \\
\hline
\end{tabular}

ALK: Anaplastic lymphoma kinase; Doc: Docetaxel; EGFR: EGF receptor; HR: Hazard ratio; m: Month; NSCLC: Non-small-cell lung cancer; OS: Overall survival; Pemb: Pembrolizumab; PFS: Progression-free survival; q2w: Every 2 week; q3w: Every 3 week; RR: response rate; TrAE: treatment related adverse events.

at a dose of 2 or $10 \mathrm{mg} / \mathrm{kg}$ of body weight $(\mathrm{mg} / \mathrm{kg})$ every 3 weeks or $10 \mathrm{mg} / \mathrm{kg}$ every 2 weeks, showed a $19.4 \%$ ORR and 12.0 months OS in the overall population. In a subgroup of patients $(\mathrm{n}=61)$ with a PD-L1 tumor proportion score of $50 \%$ or higher, the benefit was greater with an ORR of $41 \%$ [67]. According to this evidence and following Phase III randomized trial results, pembrolizumab has been recently approved as first-line treatment in patients with advanced NSCLC whose tumors have high PD-L1 expression (PD-L1 expression in $>50 \%$ of tumor cells, as assessed by the assay IHC 22C 3 pharmDx) [68] and in patients who have experienced disease progression after platinum chemotherapy, with at least $1 \%$ of PD-L1-positive tumor cells [8].

Atezolizumab is a humanized IgG1 MoAb-anti-PD-L1. Several clinical trials have demonstrated the efficacy of this agent in the treatment of NSCLC. In the first Phase I trial with an anti PD-L1 antibody, atezolizumab showed durable response in NCSCL patients [32]. In the Phase II randomized trial (POPLAR) patients with NSCLC who progressed on platinum chemotherapy and were treated with atezolizumab had a significant improvement in OS with a median of 12.6 months compared with 9.7 months of patients treated with docetaxel (hazard ratio [HR]: 0.73 ) with more benefit in patients with higher PD-L1 tumor cells expression and tumor-infiltrating inflammatory cells expression [65]. The following Phase III trial (OAK) [9] confirmed these results showing a significant reduction 


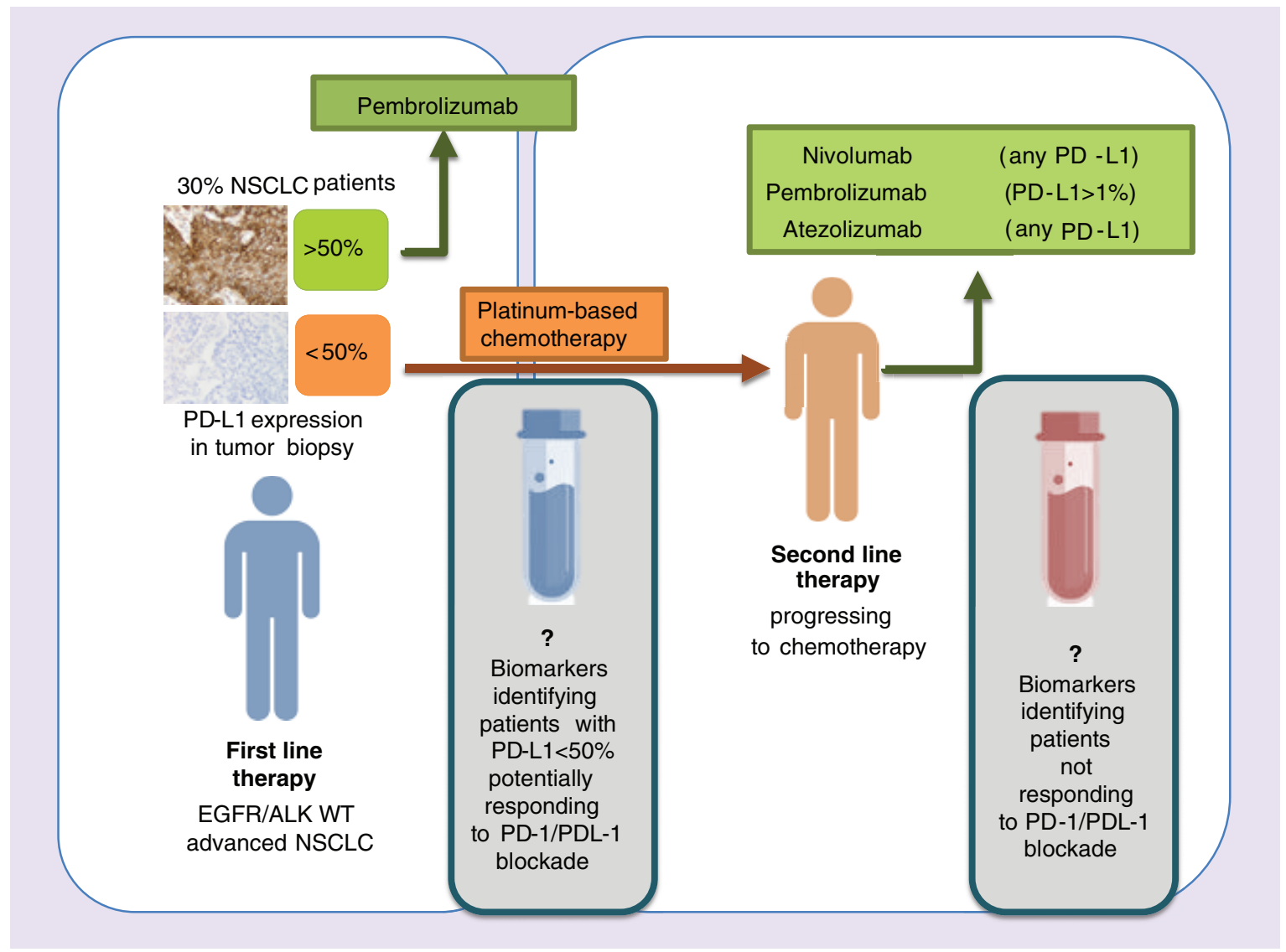

Figure 2. The clinical need of biomarkers to PD-1/PD-L1 blockade.

of death risk in the overall population, regardless of PD-L1 status, histology, age, smoking status and presence of CNS metastases, leading to the recent FDA indication for advanced NSCLC patients in second- or third-line setting.

Despite the significant survival benefit of ICIs for a large proportion of patients with advanced NSCLC, ORR is approximately $20 \%$ with a large proportion of patients not responding to these therapies $[6,7,69]$. Furthermore, a small proportion of them developed 'hyperprogression' to ICIs defined as a 'RECIST progression at the first evaluation and as a $\geq 2$-fold increase of the tumor growth rate prior and upon anti-PD-1/PD-L1 therapies' while 'early deaths' within the first 3 months of treatment with ICIs have been reported in about $10 \%$ of nonsquamous NSCLC patients included in the CheckMate 057 trial [70-72]. New clinical data are confirming the existence of this aggressive disease in a fraction of patients [70], making the identification of predictive biomarkers an urgent challenge for translational lung cancer research (Figure 2).

\section{The role of PD-L1}

Even if still limited by a lack of standardization in testing methods, the IHC assessment of tumor PD-L1 expression remains the only predictive biomarker approved for clinical setting [4]. From clinical datasets, it is becoming clearer that tumor PD-L1 expression facilitates the selection of subjects who are most likely to respond to ICIs. The Keynote 024 study clearly demonstrated that the presence of tumor PD-L1 expression more than $50 \%$ predicts pembrolizumab efficacy in first-line setting [68]. Conversely, a lower cutoff point of 5\% used in the CheckMate 026 study of nivolumab versus platinum chemotherapy failed to demonstrate any predictive role, suggesting that expanding the population assigned to ICIs may increase the risk of including patients who are less likely to benefit from PD-1 inhibition. In light of these data, the anti-PD-1 pembrolizumab is the only ICI currently approved as upfront therapy in about $30 \%$ of patients with newly diagnosed, advanced NSCLC with PD-L1 expression more than $50 \%$. Thus, the detection of PD-L1 expression on tumor cells should be always determined in order to decide 
the best upfront treatment, at least until a prospectively evaluated alternative biomarker will show similar predictive value. Differently from first-line, PD-L1 status alone is not an appropriate biomarker to exclude pretreated patients from ICIs. Indeed, the majority of trials in second-line setting showed that, even if the survival advantage obtained with anti-PD-1/PD-L1 inhibitors increased according to a higher tumor PD-L1-expression, a significant number of PD-L1-positive patients did not respond to treatment, while about 30\% of PD-L1-negative patients benefited more from ICIs as compared with standard chemotherapy with docetaxel [6-9]. These data clearly suggest that the correlation between tumor PD-L1 expression and the efficacy of these agents is imperfect, varying among different drugs and tumor subtypes, and that this could be attributed to different factors. A major issue emerging from these studies is represented by the heterogeneity of the IHC cutoffs, ranging from more than 1 to $50 \%$, as well as the multitude of detection methods based on different PD-L1 IHC MoAbs used to define positivity in tumor specimens. In this regard, the International Association for the Study of Lung CancerPathology Committee is currently working to standardize and validate a unique and reproducible IHC test for PD-L1 assessment in clinical practice [73]. The preliminary results of the International Association for the Study of Lung Cancer Blueprint Project, in line with other European projects, showed that three of four analyzed PD-L1 detection platforms had similar analytical performance, reaching an overall percentage agreement of about $90 \%$, even with some cases of discrepant results [73-75]. In addition to such technical issues, some biological aspects need to be also pointed out. Recent studies revealed that PD-L1 expression on the tumor cell surface may be regulated by two main mechanisms: the 'inflammation-driven' PD-L1 expression, consisting of an IFN- $\gamma$-mediated adaptive upregulation of PD-L1, associated with a high density of intratumor IFN-producing CD8 ${ }^{+}$T-cells, and an inflamed TME, revealing the presence of a preexisting suppressed immune response which may be reactivated by ICIs. Conversely, the 'oncogene-driven' PD-L1 expression is constitutive, not related to inflammation and associated with neither an immune response nor a T-cell tumor infiltration, thus less likely to respond to anti-PD-1/PD-L1 therapies [76,77]. A study by Ngiow et al. showed that tumors resistant to immunotherapy were infiltrated by PD-L1 ${ }^{+}$exhausted T-cells having also an overexpression of different inhibitory receptors other than PD-L1, such as TIM3, CTLA-4, LAG3 and $\mathrm{B}$ and $\mathrm{T}$ lymphocyte attenuator, and that the number of inhibitory receptors in the same $\mathrm{CD} 8^{+} \mathrm{T}$-cells can affect the severity of T-cells dysfunction [78]. Finally, it is well known that PD-L1 is a dynamic biomarker, subjected to both space (primary vs metastatic lesions) and time (interval between biopsy and subsequent treatments)dependent variability and a biopsy sample is just a snapshot of the tumor not reflecting the overall immune microenvironment [79]. Recent data revealed that multiple immune microenvironments may coexist in different metastasis within a single ovarian cancer patient [80]. In addition to intratumor heterogeneity, it should be kept in mind that the immune response is a very complex and dynamic process taking place in different sites other than TME, including lymph nodes and blood. Taken together, these data suggest that even if tumor PD-L1 expression facilitates enrichment with people who are most likely to respond to ICIs, it could not necessarily provide reliable information on the overall systemic immune status at single patient level. For this reason, the evaluation of PD-L1 status alone should not be used as negative predictor to exclude pretreated patients from ICIs, but should be always integrated with the evaluation of other potential biomarkers.

\section{Potential biomarkers emerging from translational research}

A deeper understanding of the cancer immunity cycle has allowed to elucidate the complex interactions between tumor cells and host immune system and to identify the multiple environmental factors that modulate intensity and timing of the antitumor immune response at single patient level [81], defining the so called 'cancer immunity set-point' [82], an individual immune profile which can predict response to ICIs. Beyond tumor PD-L1 expression, different clinical, pathological and molecular parameters have been explored in translational studies as predictive biomarkers for clinical use (Figure 3).

\section{Clinical factors}

In order to identify additional biomarkers to guide patients' selection, multiple clinical determinants have been extensively searched. Poorer clinical prognostic factors, such as Eastern Cooperative Oncology Group Performance Status $=1$, time since last treatment less than 3 months and progressive disease as best response to prior treatment, combined with no tumor PD-L1 expression, were initially associated with a significant higher risk of death within 3 months of treatment with nivolumab in the post hoc analysis of CheckMate 057 trial [72]. However, the same parameters were also observed in the majority of patients who were still alive after 3 months of therapy with nivolumab, hence indicating that actually we do not have reliable clinical parameters to predict clinical benefit. As 


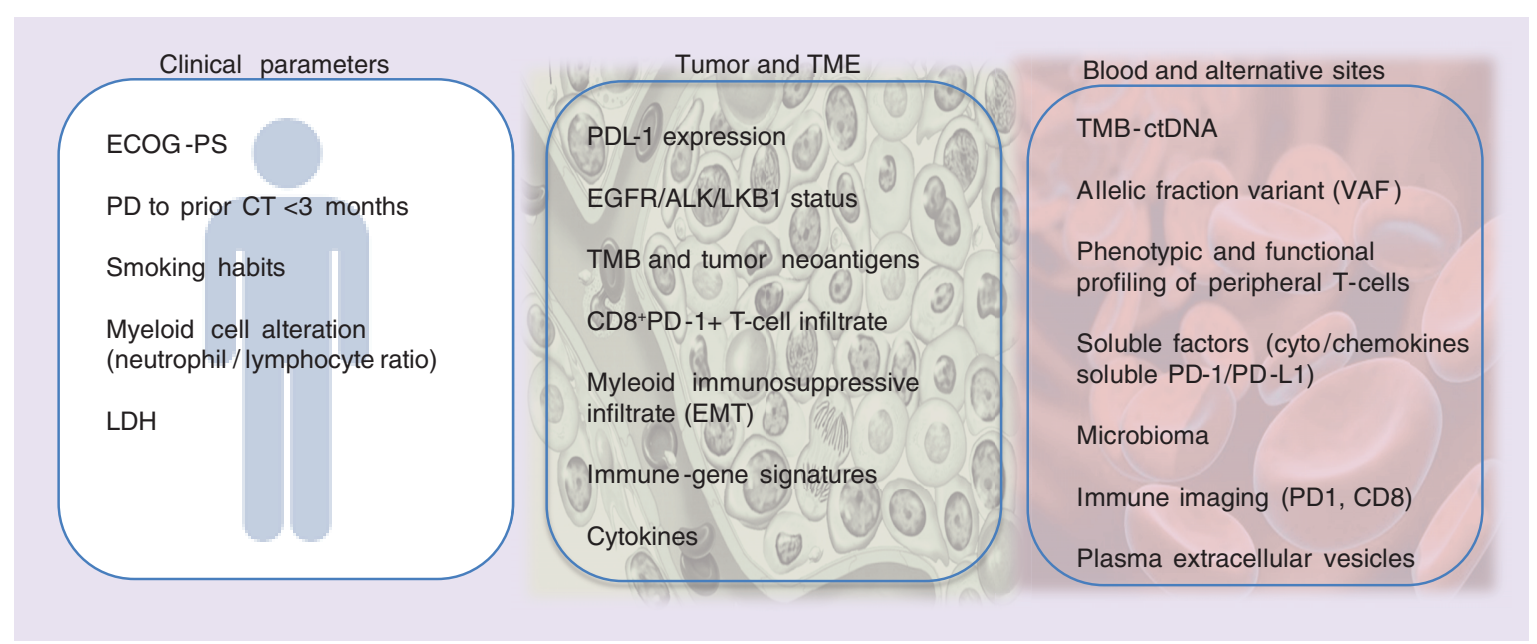

Figure 3. Predictive biomarkers to PD-1/PD-L1 blockade.

shown by randomized trials included in a recent meta-analysis ICIs prolonged survival of ever-smokers patients with advanced NSCLC patients while failed to improve survival in never-smokers, suggesting smoking status as predictive marker for clinical use [83]. Rizvi et al. have recently reported that patients with NSCLC harboring a smoking-related molecular signature had higher mutational burden and increased clinical benefit from pembrolizumab, providing the biological rationale to this clinical observation [84]. An elevated pretreatment neutrophil-to-lymphocyte ratio (NLR) may be considered as a clinical biomarker associated with poor prognosis in lung cancer [85,86], while both high baseline and follow-up NLRs are associated with progressive disease and inferior survival in NSCLC patients [87]. Some studies investigated also the prognostic/predictive role of NLR in NSCLC patients receiving immunotherapy with nivolumab, overall confirming that high pretreatment NLRs are associated with inferior outcomes and early treatment failure, regardless of other prognostic factors [88,89], while absolute lymphocyte count should not be used as criteria to exclude patients from ICIs [90]. Recently, Mezquita et al. showed that combining NLR and lactate dehydrogenase (LDH) could represent a potentially useful tool to select patients unlikely to benefit from ICI treatment [24]. Overall, these data suggest that the myeloid fraction of peripheral blood cells plays an important role in modulating antitumor response and should be further investigated as predictive biomarker of ICIs activity.

\section{Genetic alterations}

Looking for 'molecular' predictors, preclinical data showed that both the EGFR and EML/ALK oncogenic signaling in NSCLC cells could directly induce PD-L1 expression, thus suggesting that treatment with PD-1 inhibitors could be more effective in EGFR-mutated tumor models [91,92]. However, the retrospective analysis of response rate by EGFR mutation status in all the four randomized studies comparing ICIs versus docetaxel in pretreated NSCLC patients [6-9], together with a recent meta-analysis [93], have consistently demonstrated that there was no survival difference between ICIs and chemotherapy in EGFR-mutant NSCLC patients who progressed to prior EGFRtyrosine kinase inhibitors (TKI). These data point to hypothesize that patients with EGFR-mutated tumors may not be good candidates to therapy with ICIs. The discrepancy with preclinical data could stem from the very low tumor mutation burden (TMB) featuring EGFR-mutated tumors and the consequent reduced number of 'neo-antigens' triggering a protective immune response [94], as described more in details below. Hence, even if both EGFR-mutated and ALK-translocated tumors are often characterized by a significant oncogene-driven PDL1 expression [95,96], the very low rate of associated TILs makes the chances that ICIs could trigger an effective immune response rather negligible. Recently, Gainor et al. showed that tumor PD-L1 overexpression and high TILs rate were simultaneously detected in only 1/57 TKI-naive, 5/57 TKI-resistant EGFR-mutated and none of ALK-positive NSCLC patients [97]. Interesting a recent paper published in Annals of Oncology [98] analyzing the survival outcomes of 25 EGFR-mutated NSCLC patients receiving nivolumab after EGFR-TKI, revealed that T790M-negative patients received major benefit from nivolumab as compared with the T790M positive subset, likely due to the loss of the oncogene signaling. Recent findings revealed that EGFR-mutated tumors are 
associated with a high expression of the immunosuppressive molecule CD73 and reduced expression of IFN- $\gamma$ mRNA signature suggesting that the overexpression of CD73 could be responsible for the reduced activity of ICI in this subset of NSCLC [99]. According to these evidences, the preliminary results of the Phase II ATLANTIC trial evaluating the clinical activity of durvalumab in heavily pretreated $\mathrm{EGFR}^{+} / \mathrm{ALK}^{+}$NSCLC patients showed that some responses can occur also in oncogene addicted tumors who express high PD-L1 levels, however, a great and durable activity of ICI was limited to the subgroup of patients with high PD-L1 expression and EGFR/ALK wild-type [100]. Interestingly, the results of the Phase II BIRCH study have recently demonstrated a great activity of atezolizumab monotherapy as first-line treatment in PD-L1-positive NSCLC patients, regardless of tumor EGFRmutation status [101], strengthening the role of PD-L1 in first-line but also suggesting that more efforts are needed to understand the complex mechanisms modulating the antitumor immune response in oncogene-addicted NSCLC.

Beyond EGFR and ALK, a recent meta-analysis investigated the predictive role of KRAS mutations in 519 patients with previously treated NSCLC included in the CheckMate 057, OAK and POPLAR randomized studies, suggesting a greater benefit in KRAS-mutant subgroups. However, this difference did not reach statistical significance and KRAS results were available only for $17 \%$ of patients, thus not allowing to recommend KRAS as predictive biomarkers for clinical use [93]. Several preclinical and clinical evidences showed that KRAS mutations are associated with a proimmunogenic TME and clinical responses to anti-PD-1 therapies. However, KRAS-mutant NSCLC is a heterogeneous disease, including different tumor subtypes with variable biological background and clinical response to immunotherapy. A recent work revealed that tumors with co-occurring KRAS/P53 mutations were associated with higher PD-L1 expression as well as elevated $\mathrm{PD}-\mathrm{L}^{+} / \mathrm{CD}^{+}$cell ratio and increased mutation burden as compared with both KRAS or P53 single mutation. Conversely, co-occurring inactivation of liver kinase B1 (LKB1)/serine threonine kinase 11 (STK11) tumor-suppressor gene was associated with lack of tumor response and shorter progression-free survival and OS as compared with LKB1/STK11 wild-type patients with KRAS-mutant lung adenocarcinoma, suggesting LKB1-loss as genomic biomarker of innate resistance to ICIs [102]. Other papers found that loss of phosphatase and tensin homolog (PTEN) and WNT/ $\beta$-catenin signaling pathway alterations also led to primary resistance to anti-PD-1 therapy in metastatic melanoma patients who received anti-PD-1 antibodies (nivolumab and pembrolizumab), those with PTEN present tumors acquired significantly larger response than those with PTEN absent tumors. Moreover, WNT/ $\beta$-catenin signaling pathway is involved in the primary resistance to checkpoint inhibitors. Spranger $e$ al. showed in metastatic melanoma tissues that a higher CTNNB1 ( $\beta$-catenin) score correlated with the lack of T-cells $[103,104]$.

An additional mechanism of acquired resistance to ICI has been reported by Zaretsky et al. [105], who described that resistant melanoma patients may also display mutations in genes involved in the tumor sensitivity to immune response, such as, for instance, interferon-receptor-associated Janus kinase 1 and 2 (JAK1 and JAK2), or the antigen-presenting protein $\beta$-2-microglobulin. JAK1 and JAK2 mutations led to a lack of tumor response to IFN$\gamma$, with loss of its antiproliferative effects on cancer cells, while the $\beta$-2-microglobulin mutation caused the loss of surface expression of major histocompatibility complex class I (HLA-I), with the consequent lack of recognition by tumor-specific T-cells [106].

\section{Tumor mutational burden}

Several pieces of evidence have recently revealed that a high TMB is significantly associated with an increased response to immunotherapy, favoring the creation of new foreign peptides defined as neoantigens that ultimately promote the immune recognition of cancer. A recent work classified lung cancer along with melanoma among the tumors with the highest mutation burden [107], likely due to the frequent mutagen exposure represented by tobacco smoke and UV lights, respectively. Rizvi et al. first demonstrated that an elevated nonsynonymous mutation burden, including DNA repair mutations, a molecular smoking signature and a high neoantigen load, was significantly associated with clinical activity of pembrolizumab in NSCLC patients [84]. After that several papers confirmed this observation [108,109], revealing that not all the generated neoantigens are equally immunogenic, being response to immunotherapy greater in presence of clonal than subclonal neoantigens and ultimately suggesting that targeting individual tumor neoantigens could represent an innovative approach to personalize cancer immunotherapy [110]. Recently, an exploratory analysis of the CheckMate 026 trial revealed a significant superiority of nivolumab over first-line platinum chemotherapy limited to the subgroup of patients with high TMB [111]. Particularly, the analysis of TMB revealed that patients whose tumors harbor both high TMB and PD-L1 more than $50 \%$ derived the maximum benefit from ICIs. Interestingly, a great benefit was also observed in the subgroup of patients with PD-L1 less than 50\% and high TMB who would be currently excluded from ICI therapy, suggesting an additional 
predictive role for clinical setting. Furthermore, it was recently demonstrated both the feasibility and reliability of TMB analysis from the blood of NSCLC patients who received ICIs, which would be particularly useful for about $30 \%$ of patients who lack tumor tissue for molecular analysis [112]. This retrospective analysis of both POPLAR and OAK studies revealed that a high-blood TMB significantly predicted progression-free survival of pretreated NSCLC patients receiving atezolizumab, providing very interesting evidences that, if prospectively validated, will offer a valid tool for oncologists to easily monitor the efficacy of ICIs therapy. However, recent data suggest that $\mathrm{TMB}$ could be considered as an imperfect surrogate biomarker not always reflecting the overall tumor neoantigen load, because different types of molecular aberrations other than genetic mutations determine the final tumor immunogenic phenotype [113], thus explaining while tumors with a low TMB also respond to ICIs.

Loss of mutation-associated antigens may lead to acquired resistance during immunotherapy. In mouse models, elimination of mutation-associated antigens by a specific T-cell could be a mechanism of cancer immunoediting [114]. In melanoma patients who received adoptive T-cell transfer, the neoantigens recognized by T-cell are lost from tumor cells through immunoselective pressure [115]. Recent evidences showed that a potential mechanism of acquired resistance under PD-1/PD-L1 blockade therapy in initially responding NSCLC patients is represented by the loss or the reduced frequency of mutation-associated tumor neoantigens [116].

\section{Tumor lymphocyte infiltrate}

The positive prognostic value of intratumor $\mathrm{CD}^{+}$T-cells infiltrate in NSCLC has been known for several years [14]. Recent data indicate that the sensitivity to ICIs might rely on the presence of spontaneous $\mathrm{CD}^{+}{ }^{+} \mathrm{T}$-cell infiltrate expressing high levels of PD-1 and TIM3 (hot tumors) indicating the presence of a preexisting suppressed immune response that may be reactivated by anti-PD-1 agents, and ultimately suggesting an additional role as predictive biomarker. Conversely, resistance might be linked to reduced T-cell activation, defective tumor immunogenicity and a predominance of immunosuppressive cells, such as Tregs, MDSCs or secreted factors including TGF- $\beta$ or inhibitory markers in the TME (cold tumors) [77]. Both Tregs and MDSCs activity can be stimulated by IDO, which is produced by cancer cells or immune cells $[117,118]$. IDO also affects the catabolism of tryptophan and produce immunosuppressive metabolites such as kynurenine, which inhibits the proliferation of T-cells and induces T-cell anergy and apoptosis [119]. Combination of systemic inhibition of IDO and PD-1/PD-L1 blockade showed promising activity in early clinical trials in melanoma $[120,121]$ and is currently under investigation also in lung cancer. Thommen et al. studied tumor-infiltrating CD8 ${ }^{+}$T-cells of 32 patients with NSCLC and revealed that the coexpression of PD-1, TIM3, LAG3, CTLA-4, and B and T lymphocyte attenuator was associated with resistance to anti-PD-1 therapy through severe defects in T-cells cytokine production, proliferation and migration [122]. An immune evasion to anti-PD-1 therapy has been studied by Koyama et al. [123] in two fully immunocompetent mouse models of lung adenocarcinoma progressing after response to anti-PD-1 therapy. The authors observed an upregulation of alternative immune checkpoints, notably TIM3, in PD-1 antibody-bound T-cells; moreover TIM3 with other inhibitory receptors including PD-1 and LAG3 mediated T-cell exhaustion. In tumors progressing after response to anti-PD-1 therapy, and with upregulation of TIM3, they demonstrated a survival advantage with addition of a TIM3-blocking antibody following failure of PD-1 blockade, identifying a therapeutic strategy that can prolong the efficacy of immunotherapy.

Tumeh et al. performed serial biopsies from melanoma patients treated with pembrolizumab demonstrating that not only the density but also the specific intratumor location of $\mathrm{CD} 8^{+} \mathrm{T}$-cells infiltrate, as well as the simultaneous presence of high PD-L1 expression may influence the response to ICIs [54]. Subsequently, another work revealed that combining both TILs and IHC PD-L1 expression status allowed the identification of four different subgroups of TME in melanoma patients that could benefit from different treatment strategies, including combination therapies [124]. Similar findings have been also observed in NSCLC patients, where the combination of IHC PD-L1 expression and tumor-associated immune cells density identified different subsets of TME associated with distinct clinic-pathological variables and survival outcomes [125]. A recent study performed transcriptomic profiling of $\mathrm{CD}^{+} \mathrm{T}$ TILs in tumor tissue of treatment-naive lung cancer patients, revealing a wide heterogeneity in the expression of TCR and other immunotherapy targets, including PD-1, TIM3, LAG-3 and CTLA-4, which could guide the selection of different immunotherapy strategies for each patient [16]. Interestingly, this analysis identified a specific subset of enriched TILs expressing a memory cell phenotype, named as tissue-resident memory CD103 ${ }^{+}$ cells, which seem to play a key role in the T-cell-mediated response and are associated with a better survival in lung cancer patients, regardless of CD8 ${ }^{+}$T-cells tumor density. Another study characterized the different infiltrating T-cell subsets activated in both preclinical and clinical melanoma samples under ICIs therapy by mass cytometry, 
demonstrating that both anti-PD-1 and anti-CTLA- 4 agents induce the expansion of a specific exhausted-like CD8 ${ }^{+}$T-cell population while the anti-CTLA- 4 MoAbs favor also the expansion of specific ICOS+ Th1-like CD $4^{+}$ T-cells, inducing Th1 $\mathrm{CD}^{+}$immune responses and ultimately feeding the pool of short-term memory T-cells that play a key role in ICIs-induced immune response and would be otherwise destined to run out [126]. Furthermore, it allowed to identify a new subset of hyperexhausted effector T-cells that seem to be not sensitive to single-agent PD1-blockade and could likely benefit from combination strategies including CD28/B7 pathway costimulation [127]. These evidences elucidated the multiple mechanisms underlying ICIs response in the TME, thus providing the rationale for combination and personalized strategies in different tumor types, including lung cancer.

\section{Immune-gene signatures \& cytokines}

Immune-gene signatures detected both in tumor tissue and in plasma of NSCLC patients receiving ICIs have been investigated as potential surrogate of individual immune response. Particularly in an exploratory analysis of the Phase II POPLAR trial, Fehrenbacher $e$ t al. analyzed the expression profile of immune-related genes at mRNA level, showing that patients with high expression of T-effector IFN- $\Upsilon$-associated genes received major benefit from atezolizumab [65]. Similarly, NSCLC patients with high IFN- $\Upsilon$ mRNA signature in baseline tumor biopsies had greater response and longer survival to the anti-PD-L1 durvalumab, irrespectively of PD-L1 status [128]. Interestingly, a recent study in melanoma patients resistant to anti-PD-1 identified a cohort of 26 transcriptomic signatures, named 'IPRES' signature, including immunosuppressive genes (IL10, VEGFA, VEGFC), mesenchymal transition genes (AXL, ROR2, WNT5A, LOXL2, TWIST2, TAGLN, FAP), and monocyte and macrophage chemotactic genes (CCL2, CCL7, CCL8 and CCL13) commonly associated with increased hypoxia, angiogenesis, mesenchymal transition and wound healing in different cancer types such as melanoma, kidney clear cell carcinoma, colon, lung and pancreatic adenocarcinoma [129]. Other studies found that increased VEGF secretion reduces the function of effector T-cells, decreases their presence into the tumors and correlates with higher PD-1 expression on $\mathrm{CD}^{+}$T-cells [130-132]. In patients with advanced NSCLC, the combination of nivolumab with paclitaxel/carboplatin/bevacizumab revealed a good antitumor activity [133] and several ongoing trials are currently investigating the synergism of antiangiogenic and immunotherapy treatments. The TME controls T-cells migration from the circulatory system into tumors by chemokines. Th1/Tc1 responses are IFN- $\gamma$-inducible by chemokines CXCL9, CXCL10 and CXCL11 that bind CXCR3 expressed on T lymphocytes. These chemokines mediate the angiogenesis inhibition, and promote Th1-mediated immunity, inducing tumor immune-mediated destruction by recruitment of CXCR3-expressing $T$ and natural killer cells [134-136]. Low levels of CXCR3 ligands in TME reduce CD8 ${ }^{+}$T-cells infiltration that may affect the immunotherapy efficacy. A study by Peng et al. [137] showed that epigenetic silencing of Th1-type chemokines CXCL9 and CXCL10 inhibits T-cells homing. Recently, Borghaei et al. investigated the predictive role of the cytoscore, an integrated serum cytokine signature generated to quantify the effect of each identified cytokine set on OS in patients with both squamous and nonsquamous advanced NSCLC receiving nivolumab in the CheckMate 063/017/057 studies, showing that patients with a high serum cytoscore presented a significant longer OS (HR: 0.48; 95\% CI: 0.36-0.64) [138].

Overall these reports showed that the immune signatures could be used as indirect indicators of the general individual immune status, helping clinicians to select patients with a preexisting immunity who are more likely to benefit from immunotherapy. However, they require further validation in prospective studies including larger cohorts of patients. In addition to these, several other potential biomarkers are currently under investigation in translational studies and are expected to be early added to the aforementioned list.

\section{Peripheral blood biomarkers}

Most of the predictive and resistance biomarkers that are currently under evaluation or validation refer to TME and thus have to face the obvious hurdles of tumor biopsy availability and heterogeneity. As a consequence, remarkable efforts are ongoing to identify selection factors through alternative routes, relying, for instance, on blood samples, microbioma or in vivo imaging. Blood is being assessed in the context of a liquid biopsy approach, to detect tumor DNA mutations that could surrogate the role of mutational burden as source of immunogenic neoantigens. Recent studies evaluated circulating tumor (ct)DNA level changes by determining the allelic fraction variant before and after four cycles of immunotherapy showing that early ctDNA level changes could predict response/resistance to ICIs [139]. In addition to that the total number of molecular alterations detected in ctDNA could be also used to determine a 'hypermutated state' and predict immunotherapy efficacy [140]. 
The presence of circulating T-cells and antibodies specific for tumor antigens - signs of an active cellular and humoral antitumor systemic immunity - could also provide predictive information of patients responsivity. This is indicated by preliminary data showing that the level of SOX-2 (a transcriptional factor overexpressed in lung cancer)-specific T lymphocytes and antibodies identifies NSCLC patients responding to treatment with nivolumab [141]. Comparably, an early PD- ${ }^{+} \mathrm{CD} 8 \mathrm{~T}$-cell response within 4 weeks of treatment has been observed in $80 \%$ of patients with clinical benefit to anti-PD-1 therapies, while about $70 \%$ of patients with disease progression had either a delayed or absent PD-1+ CD8 T-cell response [127]. Circulating myeloid cells are also being analyzed to assess systemic immunosuppressive and inflammatory state that is expected to hamper response to immunotherapy. Despite these correlations were not always confirmed in additional studies, it can be foreseen that tumor-related T-cells and immunosuppressive responses represent a promising source of future biomarkers, given the systemic nature of the interactions that cancer cells engage with our immune defense.

\section{Microbioma}

Increasing evidence suggests that the gut microbiota promotes antitumor immunity and facilitates anti-PD-L1 efficacy. Two studies in mice showed that certain intestinal bacteria can promote antitumor immune responses and facilitate responses to immune checkpoint blockade. Sivan et al. investigated the effects of the microbiome on responses to PD-1 pathway blockade. The authors studied melanoma growth in mice with distinct commensal microbiota and analyzed differences in antitumor immunity. They observed that Bifidobacterium was present in mice responders to immunotherapy, and that oral Bifidobacterium administration alone led to tumor control, while in combination with anti PD-L1 therapy inhibited tumor growth. They showed how cancer immunotherapy response can be modulated by changing the microbiota, and that Bifidobacterium species improve DC activation and better antigen presentation with activation of $\mathrm{CD} 8^{+} \mathrm{T}$-cells [142].

Vétizou et al. studied the interaction between microbiome and responses to CTLA- 4 blockade. In mice bearing MCA205 sarcomas treated with CTLA- 4 blockade, the analyses of fecal samples revealed that treatment reduced the levels of Bacteroidales and Burkholderiales and increased the levels of Clostridiales populations. In mice and patients, T-cell response and efficacy of CTLA- 4 blockade were related specifically to B. Thetaiotaomicron or $B$. fragilis. Germ-free mice or those treated with antibiotics did not respond to CTLA-4 blockade, while germ-free mice receiving fecal transplants from patient responders to anti-CTLA-4 treatment had marked tumor responses after anti-CTLA- 4 treatment and showed colonization by $B$. fragilis. This study showed the relevant role of Bacteroidales in the immunostimulatory effects of CTLA- 4 blockade [143]. These data are interesting because of the prospect that administering a probiotic may improve antitumor immune responses during immune checkpoint blockade [144].

\section{Immune imaging}

The object of numerous researches in the field of immunocyte inhibitors is immunoimaging, in other words, the ability to visualize the distribution of immune cells on the human body in vivo through the corresponding antibodies labeled with radioactive substances that are captured by the positron emission tomography (PET) machinery to then compose tomographic images.

All these help analyze the enormous heterogeneity of tumor and immune systems and the actual distribution of immune cells involved in the various parts of the body, photographing what is the expression of the immune response in vivo.

Therefore, it is attempting to define a quantitative biomarker that can assist in the selection and treatment of patients who undergo such therapies, modulating treatment dosage and effectiveness through the dynamic and noninvasive visualization of the immune response to the drugs in question [145-147].

Murine specimens have recently been the object of the anti-PD-1 immunoblot inhibitor nivolumab, which was radiolabeled with Zirconio-89. After administration, it was possible to outline the biodistribution of tumorexpressing PD-1 tumor cells on various body sites by PET, which was subsequently verified by histological analysis $[147,148]$.

Another study in murine models, however, used another ipilimumab immunocyte inhibitor that was labeled with ${ }^{64} \mathrm{Cu}$-DOTA and CTLA-4 expression biodistribution was also detected, histologically confirmed, showing enhanced and persistent accumulation in CTLA-4-expressing tissues. Therefore, the use of a noninvasive method as the immune-PET may have an important value to assess patients' response to immunotherapy by longitudinal noninvasive monitoring of CD8 ${ }^{+} \mathrm{T}$-cells [149]. 
How to contribute to the validation of novel predictive factors: a new era for spontaneous clinical trial

The clinical development of ICIs introduced an exciting antitumor immunotherapy era. Long-term responses have been observed in multiple cancer types. Even if PD-1 or PD-L1 inhibitor monotherapy is largely well tolerated, the risk of adverse events associated with immunization increases with the use of combination regimens. Therefore, the identification of predictive biomarkers represents an urgent challenge to optimize patients' benefit, minimize the risk of toxicity and guide the combined approaches. The spotlight has been almost always on PD-L1 expression on cancer cells. Although PD-L1 positivity has been translated into a clinical benefit for this population slice, PD-L1 testing alone is insufficient for patients' selection in most cancer types, including NSCLC. There are several problems that are still pending regarding the diagnostic reliability of PD-L1 testing on cytological and small biopsy samples or liquid biopsy, tumor heterogeneity biomarkers, resistance mechanisms in positive PD-L1 tumors and responsiveness of PD-L1-negative tumor; rebiopsy to determine PD-L1 expression; heterogeneity of PD-L1 expression in primary tumor as compared with metastatic sites or after treatments. In this review, we explored emerging data on new biomarker strategies. As discussed above, there are a number of peripheral and tumor-based laboratory tests that have been screened as potential biomarkers to predict immunotherapy efficacy. Unfortunately, although some of these have been associated with positive outcomes, no one has been prospectively validated yet to allow patients' selection in clinical setting. A lot of efforts are in place to find biomarkers with satisfying predictive value that can help clinicians to select those patients who will most likely benefit from the blockade of immunological checkpoints and will get durable PD-1/PD-L1 inhibition benefits, and save resistant patients from an ineffective therapy and treatment-related toxicity. The refinement of existing biomarkers and the identification of new predictors will be key to ensuring the effective and safe use of these agents, such as PD-1/PD-L1.

The goal we have set as a group of young colleagues who mainly deal with thoracic disease in the immunotherapy era,' is promoting spontaneous translational studies characterized by a systematic collection of clinical data, tumor tissues and peripheral blood samples from NSCLC patients receiving ICIs at various medical centers for the identification of immune-related biomarkers. This analysis could also include patients' stool samples for the setup of the microbiota, which, as discussed above, is involved in immune surveillance and is known to influence immune system maturation and activity. An optimal sampling timing should always include a baseline collection before the patient undergoes any treatment; a second evaluation during the early months of therapy; and a last at the time of disease progression. This process would allow to obtain real-time and in vivo information on the treatment efficacy/resistance in NSCLC patients during the course of ICIs, providing evidence-based answers to the aforementioned clinical questions.

\section{Conclusion}

A deeper understanding of the immune landscape of cancer, including immune-evasion strategies, have led to breakthrough therapeutic advances for patients with NSCLC and have created a platform for future therapeutic developments. Immune-checkpoint inhibitors targeting PD-1/PD-L1 axis represent the current gold standard for pre-treated patients, and are now moving to first-line treatment of advanced NSCLC. With the current emphasis on precision medicine and biomarker-driven drug development, identifying predictive biomarkers remain a crucial issue in order to select those patients who may gain most benefit from immunotherapy or combination strategies.

\section{Future perspective}

Since the immune response is a very complex and dynamic spatiotemporal process, only the combination of different biological parameters integrating information from the tumor genomics and microenvironment, individual immune system and peripheral blood compartment will allow to detect the immunological profile at single patient level defining the 'cancer immunogram' [150]. Recently, Karasaki et al. performed whole exome and RNA sequencing in 20 patients with advanced NSCLC, identifying three different immunogram patterns. The 'T-cell rich' pattern, characterized by gene signatures of immune cell and checkpoint molecules, suggesting a preexisting antitumor immunity; and the T-cell poor pattern, characterized by a lack of immune cells and an intermediate pattern [151]. Similarly, Chen and Melmann described three basic immune phenotypes associated with clinical response to ICIs: the inflamed phenotype, characterized by the presence of abundant immune cells, high checkpoint protein expression and proinflammatory cytokines in the tumor, likely to respond to immunotherapy; the immune-excluded phenotype, characterized also by the presence of abundant immune cells that are confined to the stroma and do not penetrate inside the tumor; and the immune-desert phenotype characterized by paucity of immune cells both 
in the tumor and in the stroma, and presence of regulatory cells and cytokines associated with immune suppression or tolerance. Both immune-excluded and desert phenotypes may be considered as noninflamed profiles, suggesting the absence of a preexisting antitumor immunity, and rarely respond to ICIs [82]. The evaluation of patients' immunological profile at different time points during the course of ICIs therapy would allow to build the cancer immunogram as model of integrated biomarkers and ultimately overcome biological heterogeneity and personalize immunotherapy strategies. We are at the beginning of a creative period of bottom-up research activity, organized through pilot projects of increasing scope and scale, from which best practices will progressively emerge. As young oncologists, fighting lung cancer we would like to highlight that the broad current use of ICIs in clinical practice offers the unique opportunity of actively contributing to this translational research in NSCLC patients to build a bridge between research and clinical practice.

\section{Executive summary}

- In the past decade, intense research on cancer immunology allowed to elucidate the molecular basis of cancer immunogenicity and immune-editing process.

- The PD-1/PD-L1 immune checkpoint emerged as a key regulator of antitumor immune response in cancer patients.

- Systemic immunomodulatory drugs targeting the PD-1/PD-L1 axis were able to reactivate host responses and to mediate outstanding clinical benefit in a sizable proportion of patients with non-small-cell lung cancer (NSCLC).

- Immune checkpoint inhibitors, nivolumab, pembrolizumab and atezolizumab are now approved as standard treatment options in patients with advanced NSCLC.

- With the current emphasis on precision medicine, identifying predictive biomarkers remains a crucial issue in order to select the subset of patients who benefit most from immunotherapy.

- The expression PD-L1 on tumor cells should not be used as negative predictor to exclude pretreated patients from ICls; while use of pembrolizumab in first line is currently limited to NSCLC patients with tumor PD-L1 expression more than $50 \%$.

- Beyond tumor PD-L1 expression, tumor mutational burden, tumor genetic alterations, tumor lymphocyte infiltrate, inflammatory signatures and cytokines, microbioma, immune imaging and peripheral blood markers are currently investigated as predictive biomarkers for clinical use in translational research studies.

- The combination of different biological parameters integrating information from the tumor genomics and microenvironment, individual immune system and peripheral blood compartment will likely allow to define the 'cancer immunogram' at single patient level.

- The broad current use of immunotherapy in clinical practice offers the unique opportunity of actively contributing to this translational research in NSCLC patients to build a bridge between research and clinical practice.

Financial \& competing interest disclosure

The authors have no relevant affiliations or financial involvement with any organization or entity with a financial interest in or financial conflict with the subject matter or materials discussed in the manuscript. This includes employment, consultancies, honoraria, stock ownership or options, expert testimony, grants or patents received or pending, or royalties.

\section{References}

Papers of special note have been highlighted as: $\bullet$ of interest; $\bullet \bullet$ of considerable interest

1. Gardiner RE, Jahangeer S, Forde P et al. Low immunogenicity in non-small cell lung cancer; do new developments and novel treatments have a role? Cancer Metastasis Rev. 34(1), 129-144 (2015).

2. Mayekar MK, Bivona TG. Current landscape of targeted therapy in lung cancer. Clin. Pharmacol. Ther. 102(5), 757-764 (2017).

3. Cho JH. Immunotherapy for non-small-cell lung cancer: current status and future obstacles. Immune Netw. 17(6), 378-391 (2017).

4. Novello S, Barlesi F, Califano R et al. Metastatic non-small-cell lung cancer: ESMO Clinical Practice Guidelines for Diagnosis, Treatment and Follow-Up. Ann. Oncol. 27(Suppl. 5), v1-v27 (2016).

5. Brahmer JR, Horn L, Jackman D et al. Five-year follow-up from the CA209-003 study of nivolumab in previously treated advanced non-small cell lung cancer: clinical characteristics of long-term survivors. Presented at: 2017 AACR Annual Meeting. Washington, DC, USA, 1-5 April 2017.

6. Borghaei H, Paz-Ares L, Horn L et al. Nivolumab versus docetaxel in advanced nonsquamous non-small-cell lung cancer. N. Engl. J. Med. 373(17), 1627-1639 (2015). 
- It leads to approval of nivolumab in nonsquamous metastatic non-small-cell lung cancer (NSCLC).

7. Brahmer J, Reckamp KL, Baas P et al. Nivolumab versus docetaxel in advanced squamous-cell non-small-cell lung cancer. N. Engl. J. Med. 373(2), 123-135 (2015).

- It leads to approval of nivolumab in squamous metastatic NSCLC.

8. Herbst RS, Baas P, Kim DW et al. Pembrolizumab versus docetaxel for previously treated, PDL1-positive, advanced non-small-cell lung cancer (KEYNOTE-010): a randomised controlled trial. Lancet 387(10027), 1540-1550 (2016).

- It leads to approval of pembrolizumab in metastatic NSCLC with PD-L1 $>1 \%$.

9. Rittmeyer A, Barlesi F, Waterkamp D et al. Atezolizumab versus docetaxel in patients with previously treated non-small-cell lung cancer (OAK): a Phase 3, open-label, multicentre randomised controlled trial. Lancet 389(10066), 255-265 (2017).

- It leads to approval of atezolizumab in metastatic NSCLC.

10. Djureinovic D, Hallström BM, Horie M et al. Profiling cancer testis antigens in non-small-cell lung cancer. JCI Insight. 1(10), e86837 (2016).

11. Novellino L, Castelli C, Parmiani G. A listing of human tumor antigens recognized by T cells: March 2004 update. Cancer Immunol. Immunother. 54(3), 187-207 (2005).

12. Turajlic S, Litchfield $\mathrm{K}, \mathrm{Xu} \mathrm{H}$ et al. Insertion-and-deletion-derived tumour-specific neoantigens and the immunogenic phenotype: a pan-cancer analysis. Lancet Oncol. 18(8), 1009-1021 (2017).

13. Schumacher TN, Schreiber RD. Neoantigens in cancer immunotherapy. Science 348(6230), 69-74 (2015).

14. Schalper KA, Brown J, Carvajal-Hausdorf D et al. Objective measurement and clinical significance of TILs in non-small cell lung cancer. J. Natl Cancer Inst. 107(3), pii:dju 435 (2015).

15. McGranahan N, Rosenthal R, Hiley CT et al. Allele-specific HLA loss and immune escape in lung cancer evolution. Cell 171(6), 1259-1271 (2017).

16. Ganesan AP, Clarke J, Wood $\mathrm{O}$ et al. Tissue-resident memory features are linked to the magnitude of cytotoxic $\mathrm{T}$ cell responses in human lung cancer. Nat. Immunol. 18(8), 940-950 (2017).

17. Tanaka A, Sakaguchi S. Regulatory T cells in cancer immunotherapy. Cell Res. 27(1), 109-118 (2017).

18. Kargl J, Busch SE, Yang GH et al. Neutrophils dominate the immune cell composition in non-small cell lung cancer. Nat. Commun. 8, 14381 (2017)

19. Arce Vargas F, Furness AJS, Litchfield K et al. Fc effector function contributes to the activity of human anti-CTLA-4 antibodies. Cancer Cell 33(4), 649-663 (2018).

20. Lavin Y, Kobayashi S, Leader A et al. Innate immune landscape in early lung adenocarcinoma by paired single-cell analyses. Cell 169(4), 750-765 (2017).

21. Kumar V, Patel S, Tcyganov E et al. The nature of myeloid-derived suppressor cells in the tumor microenvironment. Trends Immunol. 37(3), 208-220 (2016).

22. Vetsika EK, Koinis F, Gioulbasani $\mathrm{M}$ et al. A circulating subpopulation of monocytic myeloid-derived suppressor cells as an independent prognostic/predictive factor in untreated non-small lung cancer patients. J. Immunol. Res. 2014, 659294 (2014).

23. Yin $\mathrm{Y}$, Wang J, Wang $\mathrm{X}$ et al. Prognostic value of the neutrophil to lymphocyte ratio in lung cancer: a meta-analysis. Clinics (Sao Paulo). 70(7), 524-530 (2015).

24. Mezquita L, Auclin E, Ferrara R et al. Association of the lung immune prognostic index with immune checkpoint inhibitor outcomes in patients with advanced non-small cell lung cancer. JAMA Oncol. 4(3), 351-357 (2018).

25. Barber DL, Wherry EJ, Masopust D et al. Restoring function in exhausted CD8 T cells during chronic viral infection. Nature 439(7077), 682-687 (2006).

26. Sharpe AH, Wherry EJ, Ahmed R et al. The function of programmed cell death 1 and its ligands in regulating autoimmunity and infection. Nat. Immunol. 8(3), 239-245 (2007).

27. Pardoll DM. The blockade of immune checkpoints in cancer immunotherapy. Nat. Rev. Cancer 12(4), 252-264 (2012).

28. Wherry EJ, Kurachi M. Molecular and cellular insights into T cell exhaustion. Nat. Rev. Immunol. 15(8), 486-499 (2015).

29. Brahmer JR, Drake CG, Wollner I et al. Phase I study of single-agent anti-programmed death-1 (MDX-1106) in refractory solid tumors: safety, clinical activity, pharmacodynamics, and immunologic correlates. J. Clin. Oncol. 28(19), 3167-3175 (2010).

30. Brahmer JR, Tykodi SS, Chow LQ et al. Safety and activity of anti-PDL1 antibody in patients with advanced cancer. $N$. Engl. J. Med. 366(26), 2455-2465 (2012).

31. Topalian SL, Hodi FS, Brahmer JR et al. Safety, activity, and immune correlates of anti-PD-1 antibody in cancer. N. Engl. J. Med. 366(26), 2443-2454 (2012).

-. First evidence of anti-PD-1 antibody activity in human cancers.

32. Herbst RS, Soria JC, Kowanetz M et al. Predictive correlates of response to the anti-PDL1 antibody MPDL3280A in cancer patients. Nature 515(7528), 563-567 (2014). 
33. Roychoudhuri R, Eil RL, Restifo NP. The interplay of effector and regulatory T cells in cancer. Curr. Opin. Immunol. 33, 101-111 (2015).

34. Sharma P, Allison JP. Immune checkpoint targeting in cancer therapy: toward combination strategies with curative potential. Cell 161(2), 205-214 (2015).

35. Zou W, Chen L. Inhibitory B7-family molecules in the tumour microenvironment. Nat. Rev. Immunol. 8(6), 467-477 (2008).

36. Keir ME, Butte MJ, Freeman GJ et al. PD-1 and its ligands in tolerance and immunity. Annu. Rev. Immunol. 26, 677-704 (2008).

37. Freeman GJ, Long AJ, Iwai Y et al. Engagement of the PD-1 immunoinhibitory receptor by a novel B7 family member leads to negative regulation of lymphocyte activation. J. Exp. Med. 192(7), 1027-1034 (2000).

38. Iwai $Y$, Ishida M, Tanaka $Y$ et al. Involvement of PDL1 on tumor cells in the escape from host immune system and tumor immunotherapy by PDL1 blockade. Proc. Natl Acad. Sci. USA 99(19), 12293-12297 (2002).

39. Sheppard KA, Fitz LJ, Lee JM et al. PD-1 inhibits T-cell receptor induced phosphorylation of the ZAP70/CD3zeta signalosome and downstream signaling to PKCtheta. FEBS Lett. 574(1-3), 37-41 (2004).

40. Okazaki T, Maeda A, Nishimura H et al. PD-1 immunoreceptor inhibits B cell receptor-mediated signaling by recruiting src homology 2-domain-containing tyrosine phosphatase 2 to phosphotyrosine. Proc. Natl Acad. Sci. USA 98(24), 13866-13871 (2001).

41. Fife BT, Pauken KE, Eagar TN et al. Interactions between PD-1 and PDL1 promote tolerance by blocking the TCR-induced stop signal. Nat. Immunol. 10(11), 1185-1192 (2009).

42. Park JJ, Omiya R, Matsumura Y et al. B7-H1/CD80 interaction is required for the induction and maintenance of peripheral T-cell tolerance. Blood 116(8), 1291-1298 (2010).

43. Butte MJ, Keir ME, Phamduy TB et al. Programmed death-1 ligand 1 interacts specifically with the B7-1 costimulatory molecule to inhibit T cell responses. Immunity 27(1), 111-122 (2007).

44. Wong RM, Scotland RR, Lau RL et al. Programmed death-1 blockade enhances expansion and functional capacity of human melanoma antigen-specific CTLs. Int. Immunol. 19(10), 1223-1234 (2007).

45. Fourcade J, Kudela P, Sun Z et al. PD-1 is a regulator of NY-ESO-1-specific CD8+ T cell expansion in melanoma patients. J. Immunol. 182(9), 5240-5249 (2009).

46. Matsuzaki J, Gnjatic S, Mhawech-Fauceglia P et al. Tumor-infiltrating NY-ESO-1-specific CD8+ T cells are negatively regulated by LAG-3 and PD-1 in human ovarian cancer. Proc. Natl Acad. Sci. USA 107(17), 7875-7880 (2010).

47. Wang W, Lau R, Yu D et al. PD-1 blockade reverses the suppression of melanoma antigen-specific CTL by CD4+ CD25(Hi) regulatory T cells. Int. Immunol. 21(9), 1065-1077 (2009).

48. Francisco LM, Salinas VH, Brown KE et al. PDL1 regulates the development, maintenance, and function of induced regulatory T cells. J. Exp. Med. 206(13), 3015-3029 (2009).

49. Peng W, Liu C, Xu C et al. PD-1 blockade enhances T-cell migration to tumors by elevating IFN- $\gamma$ inducible chemokines. Cancer Res. 72(20), 5209-5218 (2012).

50. Dong H, Strome SE, Salomao DR et al. Tumor-associated B7-H1 promotes T-cell apoptosis: a potential mechanism of immune evasion. Nat. Med. 8(8), 793-800 (2002).

51. Parsa AT, Waldron JS, Panner A et al. Loss of tumor suppressor PTEN function increases B7-H1 expression and immunoresistance in glioma. Nat. Med. 13(1), 84-88 (2007).

52. Marzec M, Zhang Q, Goradia A et al. Oncogenic kinase NPM/ALK induces through STAT3 expression of immunosuppressive protein CD274 (PDL1, B7-H1). Proc. Natl Acad. Sci. USA 105(52), 20852-20857 (2008).

53. Taube JM, Anders RA, Young GD et al. Colocalization of inflammatory response with B7-h1 expression in human melanocytic lesions supports an adaptive resistance mechanism of immune escape. Sci. Transl. Med. 4(127), 127ra37 (2012).

54. Tumeh PC, Harview CL, Yearley JH et al. PD-1 blockade induces responses by inhibiting adaptive immune resistance. Nature 515(7528), 568-571 (2014).

55. Ribas A. Adaptive immune resistance: how cancer protects from immune attack. Cancer Discov. 5(9), 915-919 (2015).

56. Liu Y, Zeng B, Zhang Z et al. B7-H1 on myeloid-derived suppressor cells in immune suppression by a mouse model of ovarian cancer. Clin. Immunol. 129(3), 471-481 (2008).

57. Thompson RH, Gillett MD, Cheville JC et al. Costimulatory B7-H1 in renal cell carcinoma patients: Indicator of tumor aggressiveness and potential therapeutic target. Proc. Natl Acad. Sci. USA 101(49), 17174-17179 (2004).

58. Hamanishi J, Mandai M, Iwasaki M et al. Programmed cell death 1 ligand 1 and tumor-infiltrating CD8+ T lymphocytes are prognostic factors of human ovarian cancer. Proc. Natl Acad. Sci. USA 104(9), 3360-3365 (2007).

59. Ohigashi Y, Sho M, Yamada Y et al. Clinical significance of programmed death-1 ligand-1 and programmed death-1 ligand-2 expression in human esophageal cancer. Clin. Cancer Res. 11(8), 2947-2953 (2005).

60. Wu C, Zhu Y, Jiang J et al. Immunohistochemical localization of programmed death-1 ligand-1 (PDL1) in gastric carcinoma and its clinical significance. Acta Histochem. 108(1), 19-24 (2006). 
61. Ghebeh H, Mohammed S, Al-Omair A et al. The B7-H1 (PDL1) T lymphocyte-inhibitory molecule is expressed in breast cancer patients with infiltrating ductal carcinoma: correlation with important high-risk prognostic factors. Neoplasia 8(3), 190-198 (2006).

62. Hino R, Kabashima K, Kato Y et al. Tumor cell expression of programmed cell death-1 ligand 1 is a prognostic factor for malignant melanoma. Cancer 116(7), 1757-1766 (2010).

63. Rosenwald A, Wright G, Leroy $\mathrm{K}$ et al. Molecular diagnosis of primary mediastinal B cell lymphoma identifies a clinically favorable subgroup of diffuse large B cell lymphoma related to Hodgkin lymphoma. J. Exp. Med. 198(6), 851-862 (2003).

64. Blank C, Brown I, Peterson AC et al. PDL1/B7H-1 inhibits the effector phase of tumor rejection by $\mathrm{T}$ cell receptor (TCR) transgenic CD8+ T cells. Cancer Res. 64(3), 1140-1145 (2004).

65. Fehrenbacher L, Spira A, Ballinger $\mathrm{M}$ et al. Atezolizumab versus docetaxel for patients with previously treated non-small-cell lung cancer (POPLAR): a multicentre, open-label, Phase 2 randomised controlled trial. Lancet 387(10030), 1837-1846 (2016).

66. Gettinger S, Horn L, Gandhi L et al. Overall survival and long-term safety of nivolumab (anti-programmed death 1 antibody, BMS-936558, ONO-4538) in patients with previously treated advanced non-small-cell lung cancer. J. Clin. Oncol. 33(18), 2004-2012 (2016).

67. Garon EB, Rizvi NA, Hui R et al. Pembrolizumab for the treatment of non-small-cell lung cancer. N. Engl. J. Med. 372(21), 2018-2028 (2015).

68. Reck M, Rodríguez-Abreu D, Robinson AG et al. Pembrolizumab versus chemotherapy for PDL1-positive non-small-cell lung cancer. $N$. Engl. J. Med. 375(19), 1823-1833 (2016).

- It leads to approval of pembrolizumab as first-line treatment of NSCLC with PD-L1 > 50\%.

69. Besse B, Johnson M, Janne PA et al. 16LBA Phase II, single-arm trial (BIRCH) of atezolizumab as first-line or subsequent therapy for locally advanced or metastatic PDL1-selected non-small cell lung cancer (NSCLC). Eur. J. Cancer 51(3), S717-S718 (2015).

70. Champiat S, Dercle L, Ammari S et al. Hyperprogressive disease is a new pattern of progression in cancer patients treated by anti-PD-1/PDL1. Clin. Cancer Res. 23(8), 1920-1928 (2017).

71. Kato S, Goodman A, Walavalkar V et al. Hyperprogressors after immunotherapy: analysis of genomic alterations associated with accelerated growth rate. Clin. Cancer Res. 23(15), 4242-4250 (2017).

72. Peters S, Cappuzzo F, Horn L et al. OA03.05 Analysis of early survival in patients with advanced non-squamous NSCLC treated with nivolumab vs docetaxel in CheckMate 057. J. Thorac. Oncol. 12(1), S253 (2017).

73. Hirsch FR, McElhinny A, Stanforth D et al. PDL1 immunohistochemistry assays for lung cancer: results from Phase 1 of the blueprint PDL1 IHC assay comparison project. J. Thorac. Oncol. 12(2), 208-222 (2017).

74. Scheel AH, Dietel M, Heukamp LC et al. Harmonized PDL1 immunohistochemistry for pulmonary squamous-cell and adenocarcinomas. Mod. Pathol. 29(10), 1165-1172 (2016).

75. Ratcliffe MJ, Sharpe A, Midha A et al. Agreement between programmed cell death ligand-1 diagnostic assays across multiple protein expression cutoffs in non-small cell lung cancer. Clin. Cancer Res. 23(14), 3585-3591 (2017).

76. Taube JM, Klein A, Brahmer JR et al. Association of PD-1, PD-1 ligands, and other features of the tumor immune microenvironment with response to anti-PD-1 therapy. Clin. Cancer Res. 20(19), 5064-5074 (2014).

77. Lizotte PH, Ivanova EV, Awad MM et al. Multiparametric profiling of non-small-cell lung cancers reveals distinct immunophenotypes. JCI Insight. 1(14), e89014 (2016).

78. Ngiow SF, Young A, Jacquelot $\mathrm{N}$ et al. A threshold level of intratumor CD8+ T-cell PD-1 expression dictates therapeutic response to anti-PD-1. Cancer Res. 75(18), 3800-3811 (2015).

79. Casadevall D, Clavé S, Taus Á et al. Heterogeneity of tumor and immune cell PDL1 expression and lymphocyte counts in surgical NSCLC samples. Clin. Lung Cancer 18(6), 682-691 (2017).

80. Jiménez-Sánchez A, Memon D, Pourpe S et al. Heterogeneous tumor-immune microenvironments among differentially growing metastases in an ovarian cancer patient. Cell 170(5), 927-938 (2017).

81. Chen DS, Mellman I. Oncology meets immunology: the cancer-immunity cycle. Immunity 39(1), 1-10 (2013).

82. Chen DS, Mellman I. Elements of cancer immunity and the cancer-immune set point. Nature 541(7637), 321-330 (2017).

-• It highlights the hallmarks of cancer immunity cycle.

83. Kim JH, Kim HS, Kim BJ. Prognostic value of smoking status in non-small-cell lung cancer patients treated with immune checkpoint inhibitors: a meta-analysis. Oncotarget 8(54), 93149-93155 (2017).

84. Rizvi NA, Hellmann MD, Snyder A et al. Cancer immunology. Mutational landscape determines sensitivity to PD-1 blockade in non-small cell lung cancer. Science 348(6230), 124-128 (2015).

-. First evidence on predictive role of mutational burden in NSCLC.

85. Mei Z, Shi L, Wang B et al. Prognostic role of pretreatment blood neutrophil-to-lymphocyte ratio in advanced cancer survivors: a systematic review and meta-analysis of 66 cohort studies. Cancer Treat. Rev. 58, 1-13 (2017).

86. Yu Y, Qian L, Cui J. Value of neutrophil-to-lymphocyte ratio for predicting lung cancer prognosis: a meta-analysis of 7,219 patients. Mol. Clin. Oncol. 7(3), 498-506 (2017). 
87. Derman BA, Macklis JN, Azeem MS et al. Relationships between longitudinal neutrophil to lymphocyte ratios, body weight changes, and overall survival in patients with non-small cell lung cancer. BMC Cancer 17(1), 141 (2017).

88. Diem S, Schmid S, Krapf M et al. Neutrophil-to-lymphocyte ratio (NLR) and platelet-to-lymphocyte ratio (PLR) as prognostic markers in patients with non-small cell lung cancer (NSCLC) treated with nivolumab. Lung Cancer 111, 176-181 (2017).

89. Nakao M, Muramatsu H, Kagawa Y et al. Immunological status may predict response to nivolumab in non-small cell lung cancer without driver mutations. Anticancer Res. 37(7), 3781-3786 (2017).

90. Sun R, Champiat S, Dercle L et al. Baseline lymphopenia should not be used as exclusion criteria in early clinical trials investigating immune checkpoint blockers (PD-1/PDL1 inhibitors). Eur. J. Cancer 84, 202-211 (2017).

91. Akbay EA, Koyama S, Carretero J et al. Activation of the PD-1 pathway contributes to immune escape in EGFR-driven lung tumors. Cancer Discov. 3(12), 1355-1363 (2013).

92. Ota K, Azuma K, Kawahara A et al. Induction of PDL1 expression by the EML4-ALK oncoprotein and downstream signaling pathways in non-small cell lung cancer. Clin. Cancer Res. 21(17), 4014-4021 (2015).

93. Lee CK, Man J, Lord S et al. Checkpoint inhibitors in metastatic EGFR-mutated non-small cell lung cancer-A meta-analysis. J. Thorac. Oncol. 12(2), 403-407 (2017).

94. Spigel DR, Schrock AB, Fabrizio D et al. Total mutation burden (TMB) in lung cancer (LC) and relationship with response to PD-1/PDL1 targeted therapies. J. Clin. Oncol. 34(15), 9017-9017 (2017).

95. D'Incecco A, Andreozzi M, Ludovini V et al. PD-1 and PDL1 expression in molecularly selected non-small-cell lung cancer patients. Br. J. Cancer 112(1), 95-102 (2015).

96. Schneider JS, Cambi F, Gollomp SM et al. GM1 ganglioside in Parkinson's disease: pilot study of effects on dopamine transporter binding. J. Neurol. Sci. 356(1-2), 118-123 (2015).

97. Gainor JF, Shaw AT, Sequist LV et al. EGFR mutations and ALK rearrangements are associated with low response rates to PD-1 pathway blockade in non-small cell lung cancer: a retrospective analysis. Clin. Cancer Res. 22(18), 4585-4593 (2016).

98. Haratani $\mathrm{K}$, Hayashi $\mathrm{H}$, Tanaka $\mathrm{T}$ et al. Tumor immune microenvironment and nivolumab efficacy in EGFR mutation-positive non-small-cell lung cancer based on T790M status after disease progression during EGFR-TKI treatment. Ann. Oncol. 28(7), 1532-1539 (2017).

99. Streicher T, Higgs BW, Wu S et al. Increased CD73 and reduced IFNG signature expression in relation to response rates to anti-PD-1(L1) therapies in EGFR-mutant NSCLC. J. Clin. Oncol. 35(15), 11505-11505 (2017).

100. Garassino MC, Cho B-C, Gray JE et al. Durable responses demonstrated with durvalumab in heavily pretreated patients with metastatic EGFR-mutated/ALK-positive NSCLC: ATLANTIC study results. Presented at: 2017 European Lung Cancer Conference (ELCC). Geneva, Switzerland, 5-8 May 2017.

101. Garassino MC, Rizvi N, Besse B, et al.OA03.02 - Atezolizumab as 1L therapy for advanced NSCLC in PDL1-selected patients: updated ORR, PFS and OS data from the BIRCH studyPresented atIASLC 17th World Conference on Lung CancerVienna, Austria, 4-7, December 2016.

102. Dong ZY, Zhong WZ, Zhang XC et al. Potential predictive value of TP53 and KRAS mutation status for response to PD-1 blockade immunotherapy in lung adenocarcinoma. Clin. Cancer Res. 23(12), 3012-3024 (2017).

103. Spranger S, Bao R, Gajewski TF. Melanoma-intrinsic $\beta$-catenin signalling prevents anti-tumour immunity. Nature 523(7559), 231-235 (2015).

104. Peng W, Chen JQ, Liu C et al. Loss of PTEN promotes resistance to T cell-mediated immunotherapy. Cancer Discov. 6(2), 202-216 (2016).

105. Zaretsky JM, Garcia-Diaz A, Shin DS et al. Mutations associated with acquired resistance to PD-I blockade in melanoma. N. Engl. J. Med. 375(9), 819-829 (2016).

106. Shin DS, Zaretsky JM, Escuin-Ordinas H et al. Primary resistance to PD-I blockade mediated by JAK1/2 mutations. Cancer Discov. 7(2), 188-201 (2017).

107. Alexandrov LB, Nik-Zainal S, Wedge DC et al. Signatures of mutational processes in human cancer. Nature 500(7463), 415-421 (2013).

108. McGranahan N, Furness AJ, Rosenthal R et al. Clonal neoantigens elicit $\mathrm{T}$ cell immunoreactivity and sensitivity to immune checkpoint blockade. Science 351(6280), 1463-1469 (2016).

109. Kowanetz M, Zou W, Shames D et al. Tumor mutation burden (TMB) is associated with improved efficacy of atezolizumab in $1 \mathrm{~L}$ and 2L+ NSCLC patients. J. Thorac. Oncol. 12(1), S321-S322 (2017).

110. Yarchoan M, Johnson BA 3rd, Lutz ER et al. Targeting neoantigens to augment antitumour immunity. Nat. Rev. Cancer 17(4), 209-222 (2017).

111. Carbone DP, Reck M, Paz-Ares L et al. First-Line nivolumab in stage IV or recurrent non-small-cell lung cancer. N. Engl. J. Med. 376(25), 2415-2426 (2017).

112. Gandara D. Cancer in adolescents biomarkers non-small-cell lung cancer, metastatic cancer immunology and immunotherapy lung and other thoracic tumours translational research. Ann. Oncol. 28(5), v460-v496 (2017). 
113. Turajlic S, Litchfield $\mathrm{K}, \mathrm{Xu} \mathrm{H}$ et al. Insertion-and-deletion-derived tumour-specific neoantigens and the immunogenic phenotype: a pan-cancer analysis. Lancet Oncol. 18(8), 1009-1021 (2017).

114. Matsushita H, Vesely MD, Koboldt DC et al. Cancer exome analysis reveals a T-cell-dependent mechanism of cancer immunoediting. Nature 482(7385), 400-404 (2012).

115. Verdegaal EM, de Miranda NF, Visser M et al. Neoantigen landscape dynamics during human melanoma-T cell interactions. Nature 536(7614), 91-95 (2016).

116. Anagnostou V, Smith KN, Forde PM et al. Evolution of neoantigen landscape during immune checkpoint blockade in non small cell lung cancer. Cancer Discov. 7(3), 264-276 (2017).

117. Munn DH, Mellor AL. Indoleamine 2,3 dioxygenase and metabolic control of immune responses. Trends Immunol. 34(3), 137-143 (2013).

118. Prendergast GC, Smith C, Thomas S et al. Indoleamine 2,3-dioxygenase pathways of pathogenic inflammation and immune escape in cancer. Cancer Immunol. Immunother. 63(7), 721-735 (2014).

119. Platten M, Wick W, Van den Eynde BJ. Tryptophan catabolism in cancer: beyond IDO and tryptophan depletion. Cancer Res. 72(21), 5435-5440 (2012).

120. Holmgaard RB, Zamarin D, Munn DH et al. Indoleamine 2,3-dioxygenase is a critical resistance mechanism in antitumor T cell immunotherapy targeting CTLA-4. J. Exp. Med. 210(7), 1389-1402 (2013).

121. Spranger S, Koblish HK, Horton B et al. Mechanism of tumor rejection with doublets of CTLA-4, PD-1/PDL1, or IDO blockade involves restored IL-2 production and proliferation of CD8(+) T cells directly within the tumor microenvironment. J. Immunother. Cancer 2, 3 (2014).

122. Thommen DS, Schreiner J, Müller P et al. Progression of lung cancer is associated with increased dysfunction of $\mathrm{T}$ cells defined by coexpression of multiple inhibitory receptors. Cancer Immunol. Res. 3(12), 1344-1355 (2015).

123. Koyama S, Akbay EA, Li YY et al. Adaptive resistance to therapeutic PD-I blockade is associated with upregulation of alternative immune checkpoints. Nat. Commun. 7, 10501 (2016).

124. Teng MW, Ngiow SF, Ribas A et al. Classifying cancers based on T-cell Infiltration and PDL1. Cancer Res. 75(11), 2139-2145 (2015).

125. Parra ER, Behrens C, Rodriguez-Canales J et al. Image analysis-based assessment of PDL1 and tumor-associated immune cells density supports distinct intratumoral microenvironment groups in non-small cell lung carcinoma patients. Clin. Cancer Res. 22(24), 6278-6289 (2016).

126. Wei SC, Levine JH, Cogdill AP et al. Distinct cellular mechanisms underlie anti-CTLA-4 and anti-PD-1 checkpoint blockade. Cell 170(6), 1120-1133 (2017).

127. Kamphorst AO, Wieland A, Nasti T et al. Rescue of exhausted CD8 T cells by PD-1-targeted therapies is CD28-dependent. Science 355(6332), 1423-1427 (2017).

128. Higgs W, Morehouse CA, Streicher K et al. A baseline IFNG gene expression signature correlates with clinical outcomes in durvalumab-treated advanced NSCLC cancer patients. Presented at: 2017 AACR Annual Meeting. Washington, DC, USA, 1-5 April 2017.

129. Hugo W, Zaretsky JM, Sun L et al. Genomic and transcriptomic features of response to anti-PD-1 therapy in metastatic melanoma. Cell 165(1), 35-44 (2016).

130. Motz GT, Santoro SP, Wang LP et al. Tumor endothelium FasL establishes a selective immune barrier promoting tolerance in tumors. Nat. Med. 20(6), 607-615 (2014).

131. Ohm JE, Gabrilovich DI, Sempowski GD et al. VEGF inhibits T-cell development and may contribute to tumor-induced immune suppression. Blood 101(12), 4878-4886 (2003).

132. Voron T, Colussi O, Marcheteau E et al. VEGF-A modulates expression of inhibitory checkpoints on CD8 ${ }^{+} \mathrm{T}$ cells in tumors. J. Exp. Med. 212(2), 139-148 (2015).

133. Kanda S, Goto K, Shiraishi H et al. Safety and efficacy of nivolumab and standard chemotherapy drug combination in patients with advanced non-small-cell lung cancer: a four arms Phase Ib study. Ann. Oncol. 27(12), 2242-2250 (2016).

134. Loetscher M, Gerber B, Loetscher P et al. Chemokine receptor specific for IP10 and mig: structure, function, and expression in activated T-lymphocytes. J. Exp. Med. 184(3), 963-969 (1996).

135. Moser M. Regulation of Th1/Th2 development by antigen-presenting cells in vivo. Immunobiology 204(5), 551-557 (2001).

136. Rabin RL, Park MK, Liao F et al. Chemokine receptor responses on T cells are achieved through regulation of both receptor expression and signaling. J. Immunol. 162(7), 3840-3850 (1999).

137. Peng D, Kryczek I, Nagarsheth N et al. Epigenetic silencing of TH1-type chemokines shapes tumour immunity and immunotherapy. Nature 527(7577), 249-253 (2015).

138. Borghaei H, Brahmer JR, Horn L et al. Nivolumab (nivo) vs docetaxel (doc) in patients (pts) with advanced NSCLC: CheckMate 017/057 2-y update and exploratory cytokine profile analyses. J. Clin. Oncol. 34(15), 9025-9025 (2016). 
139. Goldberg SB, Narayan A, Kole AJ et al. Early assessment of lung cancer immunotherapy response via circulating tumor DNA. Clin. Cancer Res. 24(8), 1872-1880 (2018).

140. Khagi Y, Goodman AM, Daniels GA et al. Hypermutated circulating tumor DNA: correlation with response to checkpoint inhibitor-based immunotherapy. Clin. Cancer Res. 23(19), 5729-5736 (2017).

141. Dhodapkar KM, Gettinger SN, Das R et al. SOX2-specific adaptive immunity and response to immunotherapy in non-small cell lung cancer. Oncoimmunology 2(7), e25205 (2013).

142. Sivan A, Corrales L, Hubert $\mathrm{N}$ et al. Commensal Bifidobacterium promotes antitumor immunity and facilitates anti-PDL1 efficacy. Science 350(6264), 1084-1089 (2015).

143. Vétizou M, Pitt JM, Daillère R et al. Anticancer immunotherapy by CTLA-4 blockade relies on the gut microbiota. Science 350(6264), 1079-1084 (2015).

144. Pitt JM, Vétizou M, Gomperts Boneca I et al. Enhancing the clinical coverage and anticancer efficacy of immune checkpoint blockade through manipulation of the gut microbiota. Oncoimmunology 6(1), e1132137 (2016).

145. Lamberts LE, Williams SP, Terwisscha van Scheltinga AG et al. Antibody positron emission tomography imaging in anticancer drug development. J. Clin. Oncol. 33(13), 1491-1504 (2015).

146. Gangadaran P, Ahn BC. Molecular imaging: a useful tool for the development of natural killer cell-based immunotherapies. Front. Immunol. 8, 1090 (2017).

147. Cole EL, Kim J, Donnelly DJ et al. Radiosynthesis and preclinical PET evaluation of 89Zr-nivolumab (BMS-936558) in healthy non-human primates. Bioorg. Med. Chem. 25(20), 5407-5414 (2017).

148. England CG, Jiang D, Ehlerding EB et al. 89Zr-labeled nivolumab for imaging of T-cell infiltration in a humanized murine model of lung cancer. Eur. J. Nucl. Med. Mol. Imaging 45(1), 110-120 (2018).

149. Rashidian M, Ingram JR, Dougan M et al. Predicting the response to CTLA-4 blockade by longitudinal noninvasive monitoring of CD8 T cells. J. Exp. Med. 214(8), 2243-2255 (2017).

150. Blank CU, Haanen JB, Ribas A et al. Cancer Immunology. The "cancer immunogram". Science 352(6286), 658-660 (2016).

151. Karasaki T, Nagayama K, Kuwano $\mathrm{H}$ et al. An immunogram for the cancer-immunity cycle: towards personalized immunotherapy of lung cancer. J. Thorac. Oncol. 12(5), 791-803 (2017). 